\title{
Chiral Dynamics and S-wave Contributions in Semileptonic B decays
}

\author{
Michael Döring ${ }^{a}$, Ulf-G. Meißner ${ }^{a, b}$ and Wei Wang ${ }^{a}$ \\ ${ }^{a}$ Helmholtz-Institut für Strahlen- und Kernphysik and Bethe Center \\ for Theoretical Physics, Universität Bonn, D-53115 Bonn, Germany \\ ${ }^{b}$ Institute for Advanced Simulation, Institut für Kernphysik and Jülich Center for Hadron Physics, \\ JARA-FAME and JARA-HPC, Forschungszentrum Jülich, D-52425 Jülich, Germany
}

\begin{abstract}
The flavor-changing neutral current process $b \rightarrow s l^{+} l^{-}$is beneficial to testing the standard model and hunting for new physics scenarios. In exclusive decay modes like $B \rightarrow K^{*}(892) l^{+} l^{-}$, the S-wave effects may not be negligible and thus have to be reliably estimated. Using the scalar form factors derived from dispersion relations in two channels and matched to Chiral Perturbation Theory, we investigate the S-wave contributions in $\bar{B}^{0} \rightarrow K^{-} \pi^{+} l^{+} l^{-}$, with the $K \pi$ invariant mass lying in the vicinity of the mass of $K^{*}(892)$, and the $B_{s} \rightarrow K^{-} K^{+} l^{+} l^{-}$with $m_{K K} \sim m_{\phi}$. We find that the S-wave will modify differential decay widths by about $10 \%$ in the process of $\bar{B}^{0} \rightarrow K^{-} \pi^{+} l^{+} l^{-}$and about $5 \%$ in $B_{s} \rightarrow K^{-} K^{+} l^{+} l^{-}$. A forward-backward asymmetry for the charged kaon in the final state arises from the interference between the $\mathrm{S}$-wave and $\mathrm{P}$-wave contributions. The measurement of this asymmetry offers a new way to determine the variation of the $K \pi$ S-wave phase versus the invariant mass.
\end{abstract}

PACS numbers: 13.20.He; 14.40.Be;

\section{INTRODUCTION}

One of the foremost open questions in our current knowledge of particle physics is whether new degrees of freedom are relevant for the phenomena at the $\mathrm{TeV}$ energy scale. The quest for these new particles which have distinguishable signatures compared to the standard model (SM) particles are of high priority at the ongoing collider experiments. On the other hand, low-energy processes may be influenced by them and the corresponding predictions on physical observables will be shifted away from the SM. Rare $B$ decays, with highly suppressed decay probabilities in the SM, are likely sensitive to these new degrees of freedom and therefore can be exploited as indirect searches. In particular, the flavor-changing-neutral-current processes $B \rightarrow K^{*}(892) l^{+} l^{-}$and $B_{s} \rightarrow \phi(1020) l^{+} l^{-}$can provide a wealth of information, in terms of a number of observables ranging from decay fractions, forward-backward asymmetries (FBAs), polarizations to a full angular analysis [1 3]. The recent measurements of $B \rightarrow K^{*}(892) l^{+} l^{-}$by the LHCb collaboration based on the $1 \mathrm{fb}^{-1}$ [4] data sample show no significant deviations from the SM theory [5 15$]$. This great success of the SM implies that new physics effects are likely small, and therefore renders the precision predictions for the involved quantities particularly important.

The process $B \rightarrow K^{*}(892)(\rightarrow K \pi) l^{+} l^{-}$is a quasi-four-body decay, and in principle other $K \pi$ resonant and nonresonant states may also contribute in the same final state, and thus dilute the discrimination between new physics and standard model. Theoretically, a general formula that includes various contributions has been derived in Refs. 16, 17]. In terms of helicity amplitudes, a compact form for the full angular distributions is obtained, through which the branching ratios, forward-backward asymmetries and polarizations can be easily projected. Adopting these formulas, the S-wave contribution in the $B \rightarrow K \pi l^{+} l^{-}$has been estimated in recent publications [18 20], and it is pointed out that contributions from the S-wave $K \pi$ state are not negligible and should be taken into account in future measurements. It is noticeable that in these studies, the S-wave $K \pi$ interaction is parametrized in terms of a Breit-Wigner formula, which is not justified especially for the broad scalar meson $\kappa \equiv K_{0}^{*}(800)$.

On the other hand, the scattering of light mesons are basic processes in QCD that deserve accurate measurements. In the $K \pi$ system, $S$-wave interactions proceeding through isospin $I=1 / 2$ states are of particular interest because 
they depend on the presence of scalar resonances. Studies of the scalar meson $\kappa$ can thus benefit from accurate measurements of the $I=1 / 2 S$-wave phase below $m_{K \pi}=1 \mathrm{GeV}$. The current available information on the $I=1 / 2$ $K \pi$ scattering comes from the decays $D^{+} \rightarrow K^{-} \pi^{+} e^{+} \nu_{e}$ [21] or $D \rightarrow K \pi \pi$ [22 27] as well as $J / \psi$ decays [28]. For energies beyond $\sqrt{s} \sim 1 \mathrm{GeV}$ there is the partial wave amplitude based on LASS data widely used in the literature [29]. First data from lattice calculation start to emerge [30, 31] though still at unphysical pion masses; constraints from chiral perturbation theory can be used to extract the $K \pi$ phase from the finite volume used in lattice calculations 32 34]. In the case of $K \bar{K}$, the constraint from $D_{s} \rightarrow K^{+} K^{-} e^{+} \nu_{e}$ is less precise [35], while the semileptonic $D_{s}$ decays have been used to examine the $\pi \pi$ interaction [36].

In this work, we improve the analysis in Refs. [18 20] by combining the knowledge of B meson weak decays, mainly based on operator product expansion and perturbation theory in QCD, and the low-energy effective theory for $K \pi / K \bar{K}$ interaction, namely Chiral Perturbation Theory (CHPT). CHPT can be used to constrain the form factor. Multiple subtractions for the Muskhelishvili-Omnès problem allow the systematic matching to CHPT and thus to fix the polynomial ambiguity in $s$ to a given order. We pursue this method to determine the $K \pi$ form factor by a match up to next-to-leading order. Also, we present a numerical scheme to solve the set of integral equations for cases when iteration does not converge. For the $K \bar{K}$ form factor, we rely on the model predictions of unitarized CHPT at leading order. The methodology we employ here was pioneered for B-decays in Ref. [37].

Using these results for the $K \pi / K \bar{K}$ scalar form factors and the heavy-to-light transition matrix elements calculated in the perturbative QCD approach [38 42], we study the S-wave contribution and its interference with $\mathrm{P}$-wave. We will show that the size of the S-wave pollution to the differential decay width in $B \rightarrow K^{*} l^{+} l^{-}$is about $10 \%$ while it is about $5 \%$ in $B_{s} \rightarrow \phi l^{+} l^{-}$. At last, we will discuss a subtraction method in the integration over $m_{K \pi}^{2}$ and $m_{K \bar{K}}^{2}$, which projects out the P-wave contributions and suppresses the effects of the S-wave to less than $1 \%$.

The paper is organized as follows. Sec. II recalls the differential decay distributions and the partially integrated quantities in $B \rightarrow K^{*} l^{+} l^{-}$and $B \rightarrow K_{0}^{*} l^{+} l^{-}$. In Sec. III we calculate the necessary $K^{-} \pi^{+}$and $K^{+} K^{-}$scalar form factors. Sec. IV contains our numerical predictions. We conclude in the last section. The form factors calculated in the perturbative QCD approach, the helicity decay amplitudes and normalization are relegated to the Appendices A, $\mathrm{B}$ and $\mathrm{C}$ respectively.

\section{II. $B \rightarrow K \pi l^{+} l^{-}$ANGULAR DISTRIBUTIONS}

In this section we will give the angular distributions for $B \rightarrow K^{-} \pi^{+} l^{+} l^{-}$(and $B_{s} \rightarrow K^{+} K^{-} l^{+} l^{-}$as well) with both S-wave and P-wave contributions. Throughout this work, we will consider the $\bar{B}$ meson and the pseudoscalar mesons in the final states to be charged [Neutral mesons can be treated analogously]. We adopt the convention on the kinematics in $B \rightarrow K_{J}^{*}(\rightarrow K \pi) l^{+} l^{-}$as illustrated in Fig. 1, where $K_{J}^{*}$ is a generic kaon resonance with spin $J$. In the $B$ rest frame, the $K_{J}^{*}$ flight direction is chosen as the $z$ axis. $\theta_{K}\left(\theta_{l}\right)$ is the polar angle between the $K^{-}\left(\mu^{-}\right)$ moving direction and the $z$ axis in the $K_{J}^{*}$ (lepton pair) rest frame. $\phi$ is the angle between the two decay planes.

The effective Hamiltonian for the transition $b \rightarrow s l^{+} l^{-}$

$$
\mathcal{H}_{\mathrm{eff}}=-\frac{G_{F}}{\sqrt{2}} V_{t b} V_{t s}^{*} \sum_{i=1}^{10} C_{i}(\mu) O_{i}(\mu)
$$

involves the four-quark and the magnetic penguin operators $O_{i}$, and the $C_{i}(\mu)$ are the corresponding Wilson coefficients for these local operators $O_{i}$. $G_{F}$ is the Fermi constant, $V_{t b}=0.999176$ and $V_{t s}=-0.03972$ [43] are the CKM matrix elements. The $b$ and $s$ quark masses are $m_{b}=\left(4.67_{-0.06}^{+0.18}\right) \mathrm{GeV}$ and $m_{s}=\left(0.101_{-0.021}^{+0.029}\right) \mathrm{GeV}$ [43]. The above effective 


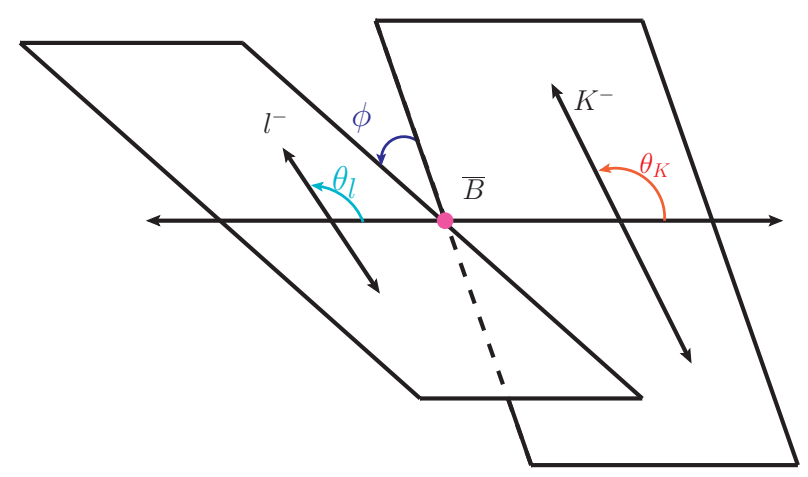

FIG. 1: Kinematics in $\bar{B} \rightarrow \bar{K}_{J}^{*}\left(\rightarrow K^{-} \pi^{+}\right) l^{+} l^{-}$. The $K_{J}^{*}$ moves along the $z$ axis in the $B$ rest frame. $\theta_{K}\left(\theta_{l}\right)$ is the angle between $z$-axis and the flight direction of $K^{-}\left(\mu^{-}\right)$in $K_{J}^{*}$ (lepton pair) rest frame, respectively. $\phi$ is the azimuth angle between the $K_{J}^{*}$ decay and lepton pair planes.

Hamiltonian results in the decay amplitude

$$
\begin{aligned}
i \mathcal{M}\left(b \rightarrow s l^{+} l^{-}\right)= & \frac{i G_{F}}{\sqrt{2}} \frac{\alpha_{\mathrm{em}}}{\pi} V_{t b} V_{t s}^{*} \times\left(\frac{C_{9}+C_{10}}{4}[\bar{s} b]_{V-A}[\bar{l} l]_{V+A}+\frac{C_{9}-C_{10}}{4}[\bar{s} b]_{V-A}[\bar{l} l]_{V-A}\right. \\
& \left.+C_{7 L} m_{b}\left[\bar{s} i \sigma_{\mu \nu}\left(1+\gamma_{5}\right) b\right] \frac{q^{\mu}}{q^{2}} \times\left[\bar{l} \gamma^{\nu} l\right]+C_{7 R} m_{b}\left[\bar{s} i \sigma_{\mu \nu}\left(1-\gamma_{5}\right) b\right] \frac{q^{\mu}}{q^{2}} \times\left[\bar{l} \gamma^{\nu} l\right]\right),
\end{aligned}
$$

where $C_{7 L}=C_{7}$ and $C_{7 R}=C_{7 L} m_{s} / m_{b}$.

The process $B \rightarrow K_{J}^{*}(\rightarrow K \pi) l^{+} l^{-}$is a four-body decay mode which undergoes two steps in the resonance picture: the $B$ meson first decays into a excited kaonic state $K_{J}^{*}$ plus a pair of leptons; the $K_{J}^{*}$ propagates followed by the strong decay into the $K \pi$. Decay amplitudes of $B \rightarrow(K \pi) l^{+} l^{-}$can be obtained by sandwiching Eq. (10) between the initial and final hadronic states, in which the spinor product $[\bar{s} b]$ will be replaced by hadronic matrix elements defined in Appendix A. The operator realization of this picture may be given as

$$
\left\langle l^{+} l^{-}|[\bar{l} l]| 0\right\rangle\left\langle K \pi|[\bar{s} b]| \bar{B}^{0}\right\rangle \simeq\left\langle l^{+} l^{-}|[\bar{l} l]| 0\right\rangle \int d^{4} p_{K_{J}^{*}} \frac{\left\langle K \pi \mid K_{J}^{*}\right\rangle\left\langle K_{J}^{*}|[\bar{s} b]| \bar{B}^{0}\right\rangle}{p_{K_{J}^{*}}^{2}-m_{K_{J}^{*}}^{2}+i m_{K_{J}^{*}} \Gamma_{K_{J}^{*}}},
$$

with $p_{K_{J}^{*}}^{2}=m_{K \pi}^{2}$. In Appendix B, we collect the required quantities in terms of helicity amplitudes that can lead to the full angular distributions

$$
\begin{aligned}
\frac{d^{5} \Gamma}{d m_{K \pi}^{2} d q^{2} d \cos \theta_{K} d \cos \theta_{l} d \phi}= & \frac{3}{8}\left[I_{1}^{c}+2 I_{1}^{s}+\left(I_{2}^{c}+2 I_{2}^{s}\right) \cos \left(2 \theta_{l}\right)+2 I_{3} \sin ^{2} \theta_{l} \cos (2 \phi)+2 \sqrt{2} I_{4} \sin \left(2 \theta_{l}\right) \cos \phi\right. \\
& +2 \sqrt{2} I_{5} \sin \left(\theta_{l}\right) \cos \phi+2 I_{6} \cos \theta_{l}+2 \sqrt{2} I_{7} \sin \left(\theta_{l}\right) \sin \phi \\
& \left.+2 \sqrt{2} I_{8} \sin \left(2 \theta_{l}\right) \sin \phi+2 I_{9} \sin ^{2} \theta_{l} \sin (2 \phi)\right] .
\end{aligned}
$$

In the massless limit for the involved leptons, and integrating over the angles $\theta_{l}, \theta_{K}$ and $\phi$, we have the dilepton mass spectrum

$$
\frac{d^{2} \Gamma}{d q^{2} d m_{K \pi}^{2}} \simeq\left|A_{L 0}^{0}\right|^{2}+\left|A_{R 0}^{0}\right|^{2}+\left|A_{L 0}^{1}\right|^{2}+\left|A_{R 0}^{1}\right|^{2}+\left|A_{L \perp}^{1}\right|^{2}+\left|A_{L||}^{1}\right|^{2}+\left|A_{R \perp}^{1}\right|^{2}+\left|A_{R \|}^{1}\right|^{2}
$$


where the functions $A_{L / R i}$ are given by

$$
\begin{aligned}
A_{L / R 0 / t} & =\sum_{J=0,1,2 \ldots} A_{L / R 0 / t}^{J} Y_{J}^{0}(\theta, 0), \\
A_{L / R \| / \perp} & =\sum_{J=0,1,2 \ldots} A_{L / R \| / \perp}^{J} Y_{J}^{-1}(\theta, 0), \\
A_{L / R 0 / t}^{J} & =\sqrt{N_{K_{J}^{*}}} \mathcal{M}_{B}\left(K_{J}^{*}, L / R, 0 / t\right) L_{K_{J}^{*}}\left(m_{K \pi}^{2}\right) \equiv\left|A_{L / R 0 / t}^{J}\right| e^{i \delta_{L / R 0 / t}^{J}}, \\
A_{L / R \| / \perp}^{J} & =\sqrt{N_{K_{J}^{*}}} \mathcal{M}_{B}\left(K_{J}^{*}, L / R, \| / \perp\right) L_{K_{J}^{*}}\left(m_{K \pi}^{2}\right) \equiv\left|A_{L / R \| / \perp}^{J}\right| e^{i \delta_{L / R \| / \perp}^{J},}
\end{aligned}
$$

with $N_{K_{J}^{*}}=\sqrt{8 / 3} \sqrt{\lambda} q^{2} \beta_{l} /\left(256 \pi^{3} m_{B}^{3}\right), \lambda \equiv\left(m_{B}^{2}-m_{K_{J}^{*}}^{2}-q^{2}\right)^{2}-4 m_{K_{J}^{*}}^{2} q^{2}$ and $\beta_{l}=\sqrt{1-4 m_{l}^{2} / q^{2}}$. The $K \pi$ lineshape is $L_{K_{J}^{*}}\left(m_{K \pi}\right)$, and for the $\mathrm{P}$-wave $K^{*}(892)$ we use the Breit-Wigner distribution:

$$
L_{K_{J}^{*}}\left(m_{K \pi}^{2}\right)=\sqrt{\frac{m_{K_{J}^{*}} \Gamma_{K_{J}^{*}}}{\pi}} \frac{1}{p_{K_{J}^{*}}^{2}-m_{K_{J}^{*}}^{2}+i m_{K_{J}^{*}} \Gamma_{K_{J}^{*}}} .
$$

The handedness label $L$ and $R$ denotes the chirality of the di-lepton system. Expressions for the weak decay amplitudes $\mathcal{M}_{B}$ can be found in Ref. [16], and to a good approximation, these amplitudes do not have large strong phases.

As a particular example, we study the angular distribution over $\theta_{K}$ :

$$
\begin{aligned}
\frac{d^{3} \Gamma}{d q^{2} d m_{K \pi}^{2} d \cos \theta_{K}} \simeq & \left\{\frac{1}{2}\left[\left|A_{L 0}^{0}\right|^{2}+\left|A_{R 0}^{0}\right|^{2}\right]+\sqrt{3} \cos \theta_{K}\left[\cos \left(\delta_{L 0}^{0}-\delta_{L 0}^{1}\right)\left|A_{L 0}^{0}\right|\left|A_{L 0}^{1}\right|+\cos \left(\delta_{R 0}^{0}-\delta_{R 0}^{1}\right)\left|A_{R 0}^{0}\right|\left|A_{R 0}^{1}\right|\right]\right. \\
& \left.+\frac{3}{2} \cos ^{2} \theta_{K}\left(\left|A_{L 0}^{1}\right|^{2}+\left|A_{R 0}^{1}\right|^{2}\right)+\frac{3}{4} \sin ^{2} \theta_{K}\left[\left|A_{L \perp}^{1}\right|^{2}+\left|A_{L \|}^{1}\right|^{2}+\left|A_{R \perp}^{1}\right|^{2}+\left|A_{R \|}^{1}\right|^{2}\right]\right\} .
\end{aligned}
$$

Compared to the distribution with only $B \rightarrow K^{*}(892) l^{+} l^{-}$, the two terms in the first line of Eq. (6) are new: the first one is the $\mathrm{S}$-wave $K \pi$ contribution, while the second term corresponds to the interference of S-wave and $\mathrm{P}$-wave. Based on this interference, one can define a forward-backward asymmetry for the charged kaon,

$$
\begin{aligned}
\frac{d^{2} A_{F B}^{K}}{d q^{2} d m_{K \pi}^{2}} & \equiv\left[\int_{0}^{1}-\int_{-1}^{0}\right] d \cos \theta_{K} \frac{d^{3} \Gamma}{d q^{2} d m_{K \pi}^{2} d \cos \theta_{K}} \\
& =\sqrt{3}\left[\cos \left(\delta_{L 0}^{0}-\delta_{L 0}^{1}\right)\left|A_{L 0}^{0}\left\|A_{L 0}^{1}\left|+\cos \left(\delta_{R 0}^{0}-\delta_{R 0}^{1}\right)\right| A_{R 0}^{0}\right\| A_{R 0}^{1}\right|\right] .
\end{aligned}
$$

The narrow-width approximation is not valid as the $\mathrm{S}$-wave $K \pi$ interaction is strong. However, the Watson theorem implies that, in the elastic regime, phases measured in $K \pi$ elastic scattering and in a decay channel in which the $K \pi$ system has no strong interaction with other hadrons are equal modulo $\pi$ radians. When the experimental data are available, this ambiguity is solved by determining the sign of the $S$-wave amplitude from data. At leading order in $\alpha_{s}$ the lepton pair $l^{+} l^{-}$indeed decouples from the $K \pi$ final state, and thus phases of B to scalar $K \pi$ decay amplitudes are equal to $I=1 / 2 K \pi$ scattering with the same isospin and angular momentum. As a consequence, we have

$$
\left\langle(K \pi)_{0}|\bar{s} \Gamma b| \bar{B}\right\rangle \propto F_{K^{-} \pi^{+}}\left(m_{K \pi}\right),
$$

with $F_{K^{-} \pi^{+}}$the scalar form factor. In this work, we will approximately use the perturbative QCD approach to compute the $B \rightarrow K_{0}^{*}$ form factor, and the line-shape is given as

$$
L_{K_{0}^{*}}^{\chi \mathrm{PT}}\left(m_{K \pi}\right)=\mathcal{N}_{\chi P T} F_{K^{-} \pi^{+}}\left(m_{K \pi}\right)
$$

with $\mathcal{N}_{\chi P T}$ being the normalization constant evaluated in Appendix $\mathbb{C}$ We will comment on the Watson theorem and the use of perturbative QCD later. 


\section{S-WAVE SCALAR FORM FACTORS}

Scalar $\pi \pi / K \bar{K}$ and $K \pi / K \eta$ form factors have been calculated within a variety of approaches using (unitarized) chiral perturbation theory [44 51] and dispersion relations [50, 52 56], in many cases using the former to constrain polynomial ambiguities of the latter. Data exist for the pion vector form factor [57 59] and the $\pi \pi$ scalar form factor (e.g., the $\phi$ in $J / \psi \rightarrow \pi \pi \phi$ acts as an isospin filter). However, the strangeness changing scalar form factor is more difficult to extract due to the mixing of $S$ and $P$ waves.

In Ref. [54], once-subtracted dispersion relations in the three channels $K \pi, K \eta$, and $K \eta^{\prime}$ were solved to determine the form factor. Here, we solve the two-channel $(K \pi, K \eta)$ problem, but with two subtractions to match not only the value but also the slope to the corresponding next-to-leading order expressions of CHPT. As it turns out, this allows for a good prediction of the known value of the form factor at the Callan-Treiman point. With an additional subtraction it would be possible to explicitly include this point as a constraint in the relations. For the one-channel case, the corresponding Omnès representation of the form factor has been formulated in Ref. 60 ].

Though inelasticities from $K \eta$ are usually taken as small in the $\kappa$ channel, the $K \eta$ channel is present. To estimate its influence, we perform a global fit to various $\pi \pi / K \bar{K}$ and $K \pi / K \eta$ scattering channels using the Inverse Amplitude Method (IAM) in the formulation of Ref. 61]. The original fit result of Ref. 61] produced an unsatisfactory description of the data in the $\kappa$ channel. The fit of the low-energy constants was improved in Ref. 32]. Here, we extend the range of data description to higher energies, to take account of the $K_{0}^{*}(1430)$, by means of a bare explicit $s$-channel resonance propagator, dressed through the couplings to the $K \pi$ and $K \eta$ channels. Coupled-channel unitarity is preserved.

The resulting amplitude is then regarded as a representation of the phase-shift data (including inelasticities) that serves as input for the Muskhelishvili-Omnès problem. For the solution of the latter we propose a numerical method to directly invert the integral equations instead of solving them by iteration. To check the influence of the inelasticity, in a second step we perform a one-channel refit to the phase-shift data, dropping the constraints from data other than the $\kappa$ channel. The one-channel problem is solved both by direct inversion and with the Omnès function.

For the $\pi \pi / K \bar{K}$ form factor evaluated in Sec. [IIB we rely on the prediction of the chiral unitary approach at order $p^{2}$, matched to the NLO expression of the form factor at order $p^{2}$. It should be noted that a corresponding procedure using the IAM in the formulation of Ref. 61], that contains also the $\mathcal{O}\left(p^{4}\right)$ contact terms, is not possible: in that formulation the method produces, almost unavoidably, spurious singularities between $s=0$ and the lowest threshold for meson-meson scattering. These singularities are then also present in the form factor.

\section{A. Scalar $K \pi$ and $K \eta$ form factors}

\section{The $K \pi$ scattering amplitude}

To obtain the coupled-channel form factor via the Muskhelishvili-Omnès relations, the scattering amplitude $T$ needs to be known, parameterized in the present study by the IAM plus a genuine resonance term,

$$
\begin{aligned}
T & =(1-V G)^{-1} V, \quad V=V_{\mathrm{IAM}}+V_{\mathrm{res}}, \\
V_{\mathrm{IAM}} & =\left(1-V^{[4]}\left(V^{[2]}\right)^{-1}\right)^{-1} V^{[2]}, \\
\left(V_{\mathrm{res}}\right)_{i j} & =\frac{g_{i} g_{j}\left[s-\left(M_{\eta}+M_{K}\right)^{2}\right]^{2}}{f_{\pi}^{2}\left(s-m_{b}\right)^{2}}
\end{aligned}
$$

with the matrices in channel space $V^{[2]}$ and $V^{[4]}$ containing the $\mathcal{O}\left(p^{2}\right)$ and $\mathcal{O}\left(p^{4}\right)$ contact interactions of Ref. [61]. An explicit expression for the scalar loop function $G$ can be found, e.g., in the Erratum of Ref. [61]. The $T$-matrix is projected to different partial waves, but the explicit resonance is only inserted in the $\kappa$ channel to take account 


\begin{tabular}{llccc}
\hline \hline \multirow{2}{*}{$\kappa(800)$} & & $z_{0}[\mathrm{MeV}]$ & $a_{-1}(K \eta)\left[M_{\pi}\right]$ & $a_{-1}(K \pi)\left[M_{\pi}\right]$ \\
& this work (2-ch.) & $792-i 279$ & $-29-i 57$ \\
& this work (1-ch.) & $715-i 283$ & $-45-i 62$ \\
& Ref. [32] ( $\chi \mathrm{U})$ & $815-i 226$ & $-30-i 57$ \\
& Ref. [66] (Roy-S.) & $658-i 279$ & $11+i 13$ \\
$K_{0}^{*}$ (1430) this work (2-ch.) & $1388-i 71$ & $-11-i 5$ & $20+i 39$ \\
& this work (1-ch.) & $1425-i 120$ & 0 & \\
& Ref. [67] (phen.) & $1427-i 135$ & & \\
\hline \hline
\end{tabular}

TABLE I: The $\kappa(800)$ and $K_{0}^{*}(1430)$ pole positions $z_{0}[\mathrm{MeV}]$ and residues $a_{-1}\left[M_{\pi}\right]$ to open channels. Uncertainties quoted in other works have been suppressed.

of the $K_{0}^{*}(1430) ; t$ and $u$-channel resonance contributions are neglected. The mixing of $\eta-\eta^{\prime}$ is neglected throughout this study; for example, in the recent study of meson-meson scattering of Ref. 62] it is taken into account. Another resonance fit to $K \pi$ has been performed in Ref. [53], see also Refs. 63, 64], and for an early study of combining CHPT with resonances, see [65]. Fit parameters of the present study are the seven low energy constants $L_{1}^{r}$ to $L_{5}^{r}$ and $L_{7}^{r}$, $L_{8}^{r}$ 61] appearing in $V^{[4]}$, as well as the two bare resonance couplings $g_{1}, g_{2}$ to the $K \pi$ and $K \eta$ channels, respectively, and the bare mass $m_{b}$.

The fit result for the considered partial waves and reaction channels is shown in Figs. 2 with the (red) solid lines and compared to the previous solution of Ref. [32] (thin dashed lines). The three new resonance parameters allow for a good data description in the energy region of the $K_{0}^{*}(1430)$. Obviously, these new parameters introduce additional freedom that is reflected in a slightly improved data description also for the other considered channels and reactions.

It should be noted that the amplitude for the $\kappa$ quantum numbers exhibits a visible cusp from the $K \eta$ channel at $E=1.043 \mathrm{GeV}$ which is an indication of considerable inelasticity. In the previous solution from Ref. [32] (thin dashed line), the cusp is even more pronounced. We accept this behavior as a consequence of the combined fit to different reactions, but will compare with a one-channel description in the following. For that, the model of Eq. (10) is reduced to the $K \pi$ channel and only the phase-shift data of the $\kappa$ channel is fitted for simplicity. This means no attempt is undertaken to determine the low-energy constants, but the fit serves merely as a one-channel representation of the phase-shift data. The result is shown with the thick dashed (blue) line in Fig. 2 .

The values of the low energy constants of the global two-channel fit are close to the ones quoted in Ref. 32]. The same applies to the pole positions of the $K^{*}(892), \rho(770), \sigma(600)$, and $f_{0}(980)$ resonances. Here, we quote only the pole positions and residues of the $\kappa(800)$ and the $K_{0}^{*}(1430)$ to open channels. As Table $\square$ shows the results for both resonances depend indeed significantly on whether a one- or a two-channel system is considered. In the two-channel (2-ch.) calculation the $\kappa(800)$ pole is at higher energies than in the one-channel calculation (1-ch.) in which case the result is closer to the Roy-Steiner determination (Roy-S.) of Ref. 66], see also Refs. 68, 69]. For the $K_{0}^{*}(1430)$, the one-channel calculation results in a broader resonance close to the one-channel phenomenological analysis (phen.) of Ref. 67], while the $K_{0}^{*}(1430)$ is much narrower in the two-channel calculation (with around $35 \%$ branching fraction into $K \eta$ ). In the latter case, the pole position is in agreement with a recent three-channel chiral unitary analysis 62 . in which a pole position of $z_{0}=1428_{-23}^{+56}-i 87_{-28}^{+53}[\mathrm{MeV}]$ is quoted. 

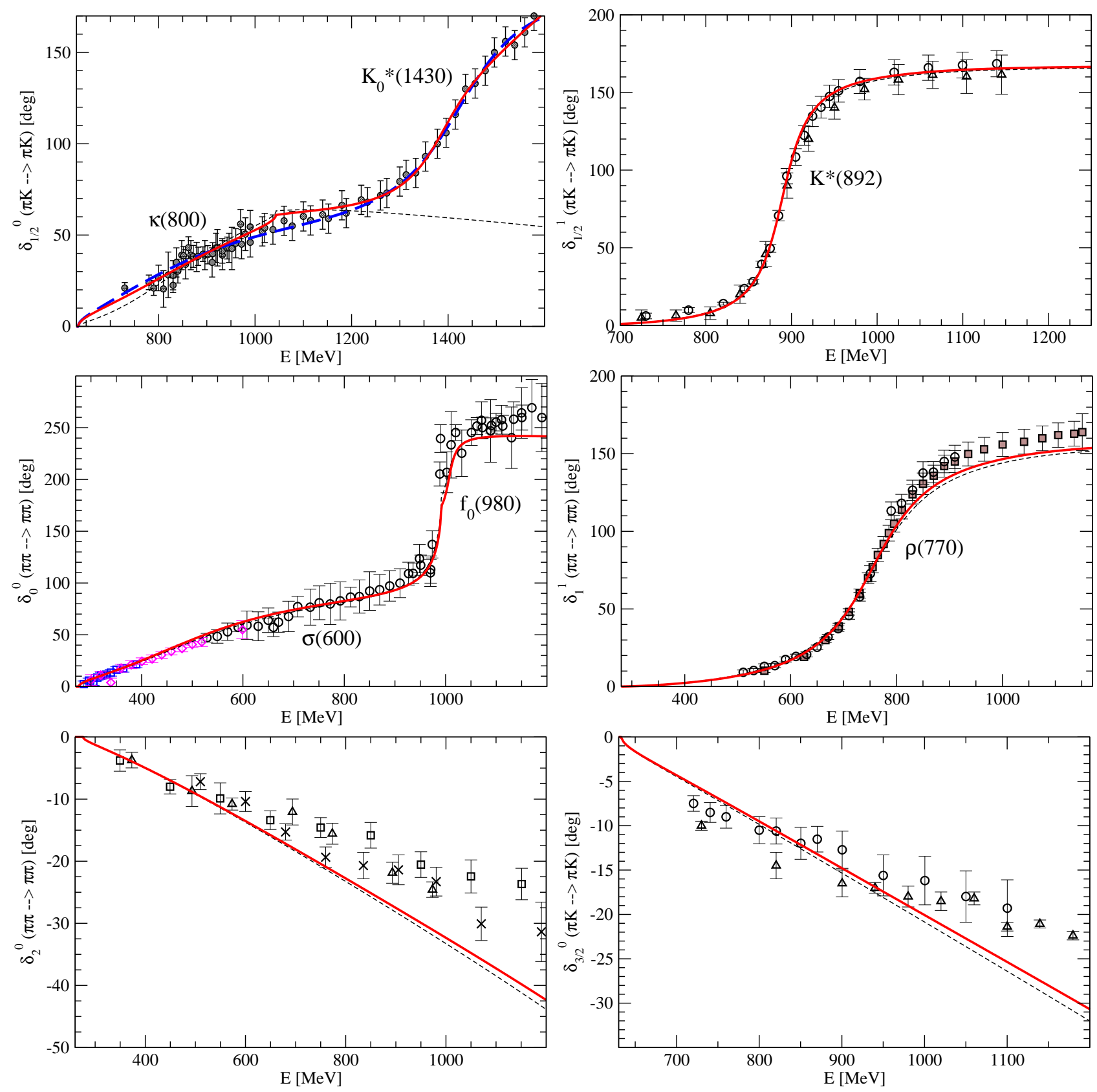

FIG. 2: Solid (red) lines: Combined coupled-channel fit to $\pi \pi$ and $K \pi$ scattering in $S$ - and $P$-waves. Thin (black) dashed lines: Results from Ref. 32]. Dashed (blue) line for the $\kappa$ channel: One-channel re-fit (only to the data in the $\kappa$ channel). Data: see Ref. 32] and references therein. The data for the $\kappa$ channel are from Ref. [61] containing the data of Refs. 29] for the higher energies.

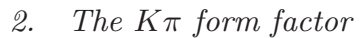

The strangeness-changing scalar form factors are defined as

$$
\langle 0|\bar{s} u| K \pi\rangle=\frac{m_{K}^{2}-m_{\pi}^{2}}{m_{s}-m_{u}} C_{X} F_{X}(s) .
$$


with $C_{X}$ being the normalization constants

$$
C_{K^{+} \pi^{0}}=\frac{1}{\sqrt{2}}, \quad C_{K^{0} \pi^{+}}=1, \quad C_{K^{+} \eta_{8}}=-\frac{1}{\sqrt{6}}, \quad C_{K^{+} \eta_{1}}=\sqrt{\frac{4}{3}} .
$$

The $K \pi$ and $K \eta_{8}$ form factors $f_{K \pi}$ and $f_{K \eta_{8}}$ have been calculated to NLO in Ref. [70]. To project to isospin $I=1 / 2$, the corresponding coefficients have to be determined. For that, we proceed in analogy to Ref. [45]. Here, the strangeness-changing combinations can be obtained from a source term

$$
\chi_{s}=2 B_{0}\left(\begin{array}{ccc}
0 & 0 & m_{\bar{u} s} \\
0 & 0 & m_{\bar{d} s} \\
m_{\bar{s} u} & m_{\bar{s} d} & 0
\end{array}\right)
$$

with the basis $(u, d, s)$. In the lowest-order chiral effective Lagrangian, the scalar source appears as

$$
\mathcal{L}=\frac{f^{2}}{4}\left\langle U^{\dagger} \chi_{s}+\chi_{s}^{\dagger} U\right\rangle
$$

and, e.g., $\bar{s} u=-\partial \mathcal{L} / \partial m_{\bar{s} u}$. To two meson fields in Eq. (14), the terms arising from all strangeness changing terms $m_{\bar{q} q^{\prime}}$ are (we quote only the sum):

$$
\begin{aligned}
\bar{q} q^{\prime}=\bar{u} s+\bar{d} s+\bar{s} u+\bar{s} d & =\frac{B_{0}}{6}\left[6\left(\pi^{-} K^{+}+K^{-} \pi^{+}+\pi^{+} K^{0}+\pi^{-} \bar{K}^{0}\right)\right. \\
& -\sqrt{2}\left(K^{-}+K^{+}\right)\left(\sqrt{3} \eta_{8}-3 \pi^{0}\right) \\
& \left.-\sqrt{2}\left(K^{0}+\bar{K}^{0}\right)\left(\sqrt{3} \eta_{8}+3 \pi^{0}\right)\right]
\end{aligned}
$$

The isospin $I=1 / 2$ combination is

$$
\left\langle 0\left|\bar{q} q^{\prime}\right| K \pi\right\rangle_{I=1 / 2}=\sqrt{\frac{3}{2}} B_{0}, \quad\left\langle 0\left|\bar{q} q^{\prime}\right| K \eta_{8}\right\rangle_{I=1 / 2}=-\frac{1}{\sqrt{6}} B_{0} .
$$

In the following, we omit an overall factor of $\sqrt{3 / 2} B_{0}$ and obtain the form factors in isospin $I=1 / 2$ as

$$
F_{K \pi}=f_{K \pi}, \quad F_{K \eta}=-\frac{1}{3} f_{K \eta_{8}}
$$

The overall normalization of the form factors is estimated in Appendix C. Recasting the expressions for $f_{K \pi}$ and $f_{K \eta_{8}}$ from Ref. [70] in terms of the leading-order meson-meson scattering transitions $K$ we obtain

$$
\begin{aligned}
& F_{K \pi}^{\chi}(s)=1+\frac{4 L_{5}^{r} s}{f^{2}}+\frac{s}{4 \Delta_{K \pi}}\left(5 \mu_{\pi}-2 \mu_{K}-3 \mu_{\eta_{8}}\right)+\bar{J}_{K \pi} K_{K \pi, K \pi}-\frac{1}{3} \bar{J}_{K \eta_{8}} K_{K \eta_{8}, K \pi} \\
& F_{K \eta_{8}}^{\chi}(s)=-\frac{1}{3}-\frac{4 L_{5}^{r} s}{3 f^{2}}-\frac{3 s}{4 \Delta_{K \pi}}\left(\mu_{\pi}-2 \mu_{K}+\mu_{\eta_{8}}\right)+\bar{J}_{K \pi} K_{K \pi, K \eta_{8}}-\frac{1}{3} \bar{J}_{K \eta_{8}} K_{K \eta_{8}, K \eta_{8}}
\end{aligned}
$$

where

$$
\begin{aligned}
& K_{K \pi, K \pi}=-\frac{1}{8 f^{2}}\left(2 \Sigma-5 s+\frac{3 \Delta_{K \pi}^{2}}{s}\right), \quad K_{K \pi, K \eta_{8}}=-\frac{1}{8 f^{2}}\left(3 s-2 \Sigma-\frac{\Delta_{K \pi}^{2}}{s}\right) \\
& K_{K \eta_{8}, K \eta_{8}}=-\frac{1}{24 f^{2}}\left(9 s+\frac{\Delta_{K \pi}^{2}}{s}-18 M_{\eta_{8}}^{2}-2 M_{K}^{2}\right)
\end{aligned}
$$

and $K_{K \eta_{8}, K \pi}=K_{K \pi, K \eta_{8}}$ due to time reversal invariance, $\Sigma=M_{\pi}^{2}+M_{K}^{2}, \Delta_{K \pi}=M_{K}^{2}-M_{\pi}^{2}$ and

$$
\begin{aligned}
\bar{J}= & \frac{1}{32 \pi^{2}}\left[2+\left(\frac{M_{1}^{2}-M_{2}^{2}}{s}-\frac{M_{1}^{2}+M_{2}^{2}}{M_{1}^{2}-M_{2}^{2}}\right) \log \frac{M_{2}^{2}}{M_{1}^{2}}-\frac{\lambda(s)}{s}\left(\log \left(s+\lambda(s)+M_{1}^{2}-M_{2}^{2}\right)+\log \left(s+\lambda(s)-M_{1}^{2}+M_{2}^{2}\right)\right.\right. \\
& \left.\left.-\log \left(-s+\lambda(s)-M_{1}^{2}+M_{2}^{2}\right)-\log \left(-s+\lambda(s)+M_{1}^{2}-M_{2}^{2}\right)\right)\right] .
\end{aligned}
$$


Note the convention $V^{[2]}=-K$ with $V^{[2]}$ from Eq. (10). The constant $f$ is taken to equal the pion decay constant $f_{\pi}=92.4 \mathrm{MeV}, \lambda^{2}(s)=\left[s-\left(M_{1}+M_{2}\right)^{2}\right]\left[s-\left(M_{1}+M_{2}\right)^{2}\right]$, and $s \equiv s+i \epsilon$ ensures that the correct sheet of the logarithm is taken. The expressions for the logarithms $\mu_{i}$ generated by chiral tadpoles in the NLO scalar form factors are given by

$$
\mu_{i}=\frac{M_{i}^{2}}{32 \pi^{2} f^{2}} \log \left(\frac{M_{i}^{2}}{\mu^{2}}\right) .
$$

For the low-energy constant $L_{5}^{r}$ we take the value from Ref. [71] of the fit $A l l p^{4}, 10^{3} \cdot L_{5}^{r}\left(\mu=M_{\rho}\right)=1.21$. In the present calculation the scale is set by the cutoff of the scalar loop function $G$ in Eq. (10) of $\Lambda=1 \mathrm{GeV}$. The scale can be calculated according to Ref. [61]

$$
\mu^{\prime}=\frac{2 \Lambda}{\sqrt{e}}
$$

up to order $\mathcal{O}\left(M_{i}^{2} / \Lambda^{2}\right)$. This expression also holds for loops with unequal masses, as we have checked explicitly. The value of the low energy constant at this $\mu^{\prime}$ is obtained by evolving $L_{5}^{r}$ from $\mu=M_{\rho}$ using the $\beta$-function [72],

$$
L_{5}^{r}(\mu)-L_{5}^{r}\left(\mu^{\prime}\right)=\frac{3}{8(4 \pi)^{2}} \log \frac{\mu^{\prime}}{\mu},
$$

resulting in $10^{3} \cdot L_{5}\left(\mu^{\prime}=1.213 \mathrm{GeV}\right)=0.131$.

\section{Matching to CHPT by twice-subtracted dispersion relations}

We write the twice-subtracted Muskhelishvili-Omnès problem for the two channels $K \pi$ and $K \eta$ as

$$
\begin{aligned}
& F_{K \pi}(s)=F_{K \pi}^{\chi}(0)+\left(F_{K \pi}^{\chi}\right)^{\prime}(0) s+\frac{s^{2}}{\pi} \int_{s_{K \pi}}^{\infty} d s^{\prime} \frac{F_{K \pi}\left(s^{\prime}\right) \sigma_{K \pi}\left(s^{\prime}\right) T_{K \pi, K \pi}^{*}\left(s^{\prime}\right)}{s^{\prime 2}\left(s^{\prime}-s-i \epsilon\right)}+\frac{s^{2}}{\pi} \int_{s_{K \eta}}^{\infty} d s^{\prime} \frac{F_{K \eta}\left(s^{\prime}\right) \sigma_{K \eta}\left(s^{\prime}\right) T_{K \eta, K \pi}^{*}\left(s^{\prime}\right)}{s^{\prime 2}\left(s^{\prime}-s-i \epsilon\right)} \\
& F_{K \eta}(s)=F_{K \eta}^{\chi}(0)+\left(F_{K \eta}^{\chi}\right)^{\prime}(0) s+\frac{s^{2}}{\pi} \int_{s_{K \pi}}^{\infty} d s^{\prime} \frac{F_{K \pi}\left(s^{\prime}\right) \sigma_{K \pi}\left(s^{\prime}\right) T_{K \eta, K \pi}^{*}\left(s^{\prime}\right)}{s^{\prime 2}\left(s^{\prime}-s-i \epsilon\right)}+\frac{s^{2}}{\pi} \int_{s_{K \eta}}^{\infty} d s^{\prime} \frac{F_{K \eta}\left(s^{\prime}\right) \sigma_{K \eta}\left(s^{\prime}\right) T_{K \eta, K \eta}^{*}\left(s^{\prime}\right)}{s^{\prime 2}\left(s^{\prime}-s-i \epsilon\right)}
\end{aligned}
$$

with $T$ from Eq. (10) and ${ }^{1} \sigma=-q_{\text {c.m. }} /(8 \pi \sqrt{s})$. The subtractions ensure that the scalar form factors $F_{K \pi}$ and $F_{K \eta}$ match the size and slope of the next-to-leading order chiral result from Eq. (18) at $s=0$.

The system of integral equations (24) cannot be solved by iteration as it is possible in other cases [54, 73]. Due to the two subtractions, this procedure is not convergent. However, it can be solved by matrix inversion. The derivation of the solution is shown for the one-channel case and then generalized to the two-channel case. We rewrite the twice subtracted dispersion relation to numerically regularize the singularity $(s \equiv s+i \epsilon)$,

$$
F(s)=F^{\chi}(0)+\left(F^{\chi}\right)^{\prime}(0) s+K(s, s) F(s)\left[\log \left(\frac{s_{\text {cut }}-s}{s}\right)+i \pi\right]+\int_{0}^{s_{\text {cut }}} d s^{\prime} \frac{K\left(s, s^{\prime}\right) F\left(s^{\prime}\right)-K(s, s) F(s)}{s^{\prime}-s-i \epsilon}
$$

with $s_{\text {cut }} \rightarrow \infty$ and the kernel

$$
K\left(s, s^{\prime}\right)=\frac{s^{2}}{\pi} \frac{\sigma\left(s^{\prime}\right) T^{*}\left(s^{\prime}\right)}{s^{\prime 2}} \Theta\left(s^{\prime}-s_{\text {thres. }}\right) .
$$

\footnotetext{
1 The matrix $T$ is defined with opposite sign in Ref. [54 which is here absorbed in the sign of $\sigma$.
} 
Adding and subtracting the singularity in this way ensures that for $s>s_{\text {thres. }}$ the integral is regular and the imaginary part is correctly evaluated while for $s<s_{\text {thres. }}$ these extra terms are absent. Additionally, the lower integration limit has been moved to the definition of the kernel $K$, the reason for which will become clearer in the following.

For the numerical evaluation, the integral is replaced by a sum according to $\int d s^{\prime} f\left(s^{\prime}\right) \rightarrow \sum_{j} w_{j} f\left(s_{j}^{\prime}\right)$ with integration weights $w_{j}$ at $s^{\prime}=s_{j}^{\prime}$. To apply the matrix inversion technique, one has to choose $s_{j}=s_{j}^{\prime}, \forall j=1, \ldots, n$, such that Eq. (25) is rewritten in discretized form as

$$
\begin{aligned}
F\left(s_{i}\right) & =F^{\chi}(0)+\left(F^{\chi}\right)^{\prime}(0) s_{i}+K\left(s_{i}, s_{i}\right)\left[\log \left(\frac{s_{\mathrm{cut}}-s_{i}}{s_{i}}\right)+i \pi\right] \\
& +w_{i} \frac{K\left(s_{i}, s_{i+1}\right) F\left(s_{i+1}\right)-K\left(s_{i}, s_{i}\right) F\left(s_{i}\right)}{s_{i+1}-s_{i}}+\sum_{j \neq i}^{n} w_{j} \frac{K\left(s_{i}, s_{j}\right) F\left(s_{j}\right)-K\left(s_{i}, s_{i}\right) F\left(s_{i}\right)}{s_{j}-s_{i}} \quad \forall i=1, \ldots, n .
\end{aligned}
$$

The sum over the integration weights has been split into a regular part $i \neq j$, and the term $i=j$ has been replaced by the right-hand derivative,

$$
\lim _{s^{\prime} \rightarrow s}\left(\frac{K\left(s, s^{\prime}\right) F\left(s^{\prime}\right)-K(s, s) F(s)}{s^{\prime}-s}\right) \rightarrow \frac{K\left(s_{i}, s_{i+1}\right) F\left(s_{i+1}\right)-K\left(s_{i}, s_{i}\right) F\left(s_{i}\right)}{s_{i+1}-s_{i}} .
$$

For the case $i=n$, this term is simply set to zero which induces an error that vanishes for sufficiently many integration points; furthermore, the derivative should be small for large $s$. One could introduce here a left-hand derivative, but this unnecessarily complicates the equations. Introducing

$$
M_{i j}= \begin{cases}\frac{K\left(s_{i}, s_{j}\right)}{s_{j}-s_{i}} w_{j} & \text { for } j \neq i, j \neq i+1, \\ \frac{K\left(s_{i}, s_{i+1}\right)}{s_{i+1}-s_{i}}\left(w_{i}+w_{i+1}\right) & \text { for } j=i+1, i \neq n, \\ K\left(s_{i}, s_{i}\right)\left[\log \left(\frac{s_{\mathrm{cut}}-s_{i}}{s_{i}}\right)+i \pi-\frac{w_{i}}{s_{i+1}-s_{i}}-\sum_{j \neq i} \frac{w_{j}}{s_{j}-s_{i}}\right] & \text { for } i=j \neq n, \\ K\left(s_{i}, s_{i}\right)\left[\log \left(\frac{s_{\mathrm{cut}}-s_{i}}{s_{i}}\right)+i \pi-\sum_{j \neq i} \frac{w_{j}}{s_{j}-s_{i}}\right] & \text { for } i=j=n,\end{cases}
$$

and the vector

$$
\mathbf{F}^{\chi}=\left(F^{\chi}(0)+\left(F^{\chi}\right)^{\prime}(0) s_{1}, \ldots, F^{\chi}(0)+\left(F^{\chi}\right)^{\prime}(0) s_{n}\right)^{T},
$$

Eq. (27) is rewritten as

$$
\mathbf{F}=\mathbf{F}^{\chi}+\mathbf{M F}
$$

that can be inverted to yield the form factor

$$
\mathbf{F}=(\mathbb{1}-\mathbf{M})^{-1} \mathbf{F}^{\chi}
$$

The scheme presented here resembles the Haftl-Tabakin scheme used for the solution of scattering problems [74]. However, the present scheme is more involved, because one has explicit singularities for $s=s^{\prime}$. As shown, those can be handled by using the numerical derivative of the integrand. In the context of a dispersive analysis of the scalar form factor of the nucleon, a similar method has been derived in Ref. 75].

The generalization to two or more channels is straightforward. For this, we introduce the channel indices $\mu, \nu$ in the kernel

$$
K_{\mu \nu}\left(s, s^{\prime}\right)=\frac{s^{2}}{\pi} \frac{\sigma_{\nu}\left(s^{\prime}\right) T_{\nu \mu}^{*}\left(s^{\prime}\right)}{s^{\prime 2}} \Theta\left(s^{\prime}-s_{\text {thres. } \nu}\right)
$$



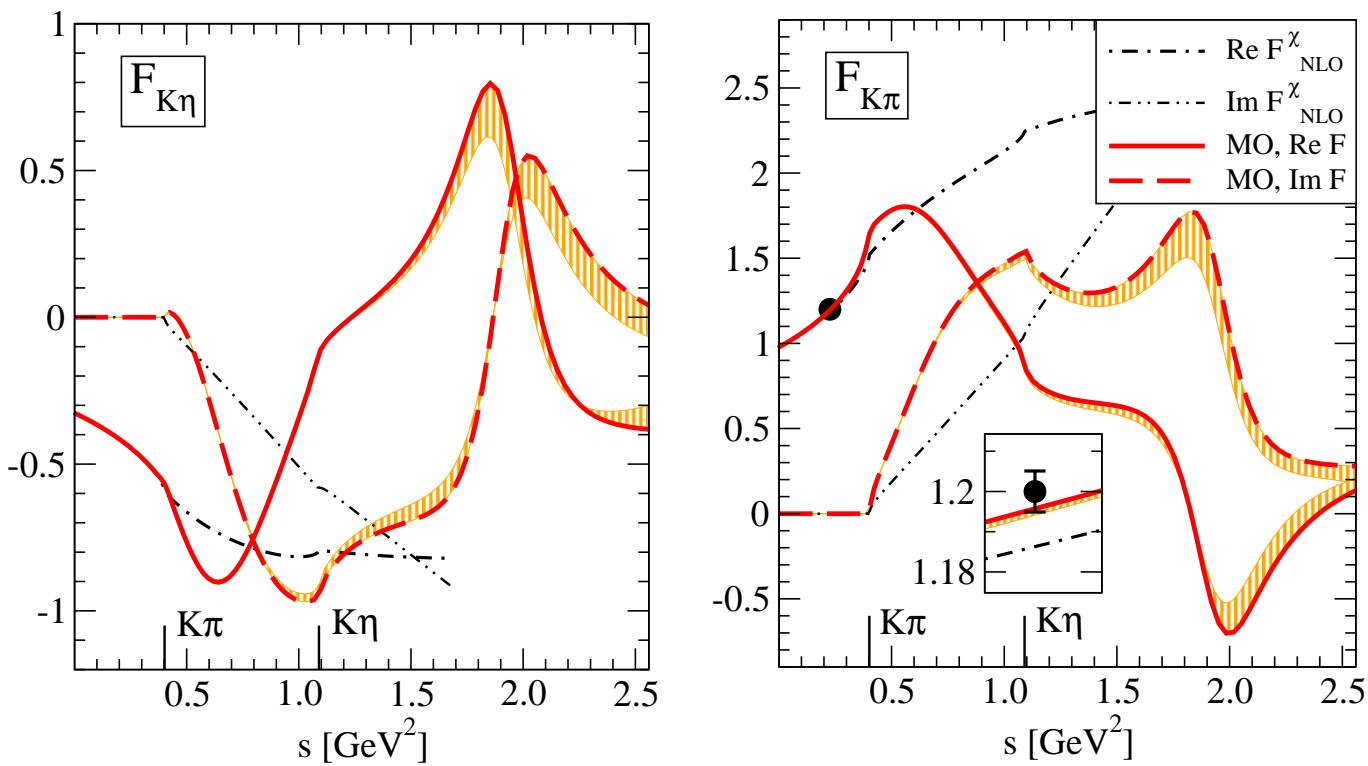

FIG. 3: The $K \eta$ (left panel) and $K \pi$ (right panel) form factors. Thick (red) lines: Muskhelishvili-Omnès solution according to Eq. (24), obtained with Eq. (34). Also, the uncertainties from higher energy input are shown (hatched areas). Dash-dotted lines: chiral next-to-leading order result [70] according to Eq. (18). The data point shows the value at the Callan-Treiman point (not included as a constraint).

that lead to a $2 n \times 2 n$ matrix $\mathbf{M}_{\mu \nu}$ according to Eq. (29). The initial coupled-channel problem of Eq. (24) can finally be rewritten as

$$
\left(\begin{array}{l}
\mathbf{F}_{1} \\
\mathbf{F}_{2}
\end{array}\right)=\left(\begin{array}{l}
\mathbf{F}_{1}^{\chi} \\
\mathbf{F}_{2}^{\chi}
\end{array}\right)+\left(\begin{array}{ll}
\mathbf{M}_{11} & \mathbf{M}_{12} \\
\mathbf{M}_{21} & \mathbf{M}_{22}
\end{array}\right)\left(\begin{array}{l}
\mathbf{F}_{1} \\
\mathbf{F}_{2}
\end{array}\right)
$$

where 1 stands for the $K \pi$ channel and 2 for the $K \eta$ channel. The solution for the $2 n$ elements $\left(F_{K \pi}\left(s_{1}\right), \ldots, F_{K \pi}\left(s_{n}\right), F_{K \eta}\left(s_{1}\right), \ldots, F_{K \eta}\left(s_{n}\right)\right)^{T}$ is then obtained by matrix inversion as before ${ }^{2}$.

\section{Results for the form factors}

With the $T$ matrix of Eq. (10) and the chiral form factors of Eq. (18) as input, the solution of Eqs. (24) is shown in Fig. [3 for $s_{\text {cut }}=(2.05 \mathrm{GeV})^{2}$. The error bands are obtained by varying $s_{\text {cut }}$ from this value down to $s_{\text {cut }}=(1.65 \mathrm{GeV})^{2}$ to show the sensitivity to the high-energy input. As the figure shows, values and slopes of the chiral results $F_{K \pi}^{\chi}(0)$ and $F_{K \eta}^{\chi}(0)$ are matched. We have checked that $F_{K \pi}$ fulfills Watson's theorem between the $K \pi$ and the $K \eta$ threshold. An additional piece of information is given by the value of the form factor at the Callan-Treiman point [55, 60], $F_{K \pi}\left(\Delta_{K \pi}\right)=1.2346(53) F_{K \pi}(0)$. It is possible to explicitly include this value in the Omnès representation of the form factor [60], fixing not only its value and slope at $s=0$ but also the curvature. In the inset of Fig. 3, we simply show our prediction of the value.

\footnotetext{
2 It finally becomes also clear why we have extended the lower integration limit to zero in writing Eq. 25). In this way, coupled-channel problems with different thresholds can be treated easily, and, second, the solution obtained for the form factors extends down to $s=0$.
} 


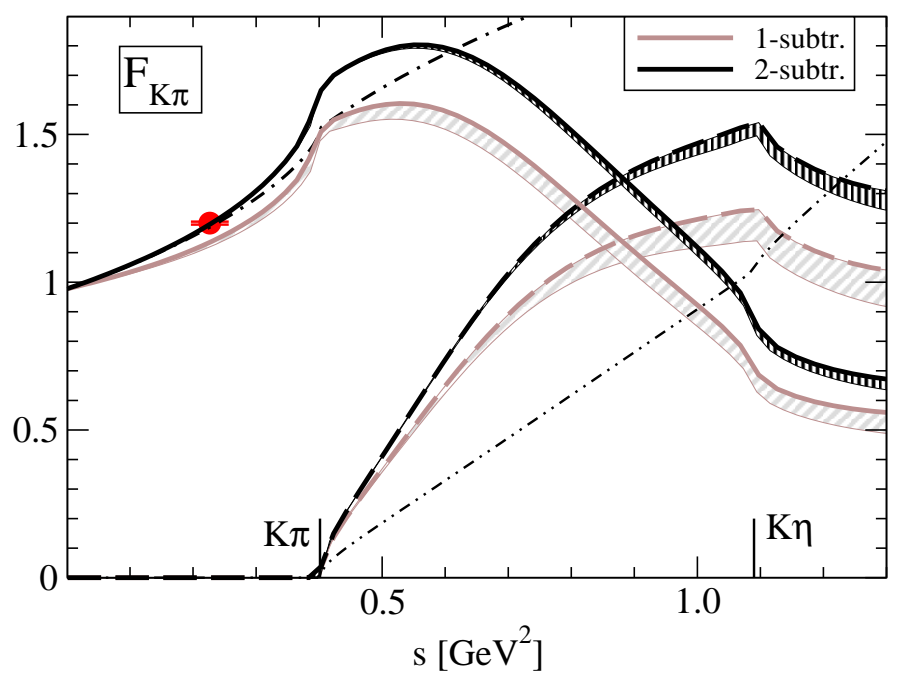

FIG. 4: Comparison of the once-subtracted Muskhelishvili-Omnès solution (faint gray lines) with the twice-subtracted result of Fig. 3 (black lines). Other curves as in Fig. 3 For the once-subtracted solution, the slope at $s=0$ is not matched and uncertainties from higher-energy input (shaded areas) are larger.

In Fig. 4 we compare to the once-subtracted version of the Muskhelishvili-Omnès problem, given by

$$
\begin{aligned}
& F_{K \pi}(s)=F_{K \pi}^{\chi}(0)+\frac{s}{\pi} \int_{s_{K \pi}}^{\infty} d s^{\prime} \frac{F_{K \pi}\left(s^{\prime}\right) \sigma_{K \pi}\left(s^{\prime}\right) T_{K \pi, K \pi}^{*}\left(s^{\prime}\right)}{s^{\prime}\left(s^{\prime}-s-i \epsilon\right)}+\frac{s}{\pi} \int_{s_{K \eta}}^{\infty} d s^{\prime} \frac{F_{K \eta}\left(s^{\prime}\right) \sigma_{K \eta}\left(s^{\prime}\right) T_{K \eta, K \pi}^{*}\left(s^{\prime}\right)}{s^{\prime}\left(s^{\prime}-s-i \epsilon\right)}, \\
& F_{K \eta}(s)=F_{K \eta}^{\chi}(0)+\frac{s}{\pi} \int_{s_{K \pi}}^{\infty} d s^{\prime} \frac{F_{K \pi}\left(s^{\prime}\right) \sigma_{K \pi}\left(s^{\prime}\right) T_{K \eta, K \pi}^{*}\left(s^{\prime}\right)}{s^{\prime}\left(s^{\prime}-s-i \epsilon\right)}+\frac{s}{\pi} \int_{s_{K \eta}}^{\infty} d s^{\prime} \frac{F_{K \eta}\left(s^{\prime}\right) \sigma_{K \eta}\left(s^{\prime}\right) T_{K \eta, K \eta}^{*}\left(s^{\prime}\right)}{s^{\prime}\left(s^{\prime}-s-i \epsilon\right)} .
\end{aligned}
$$

The solution is obtained by the same method as for the twice subtracted relations, with a modified inhomogeneity

$$
\mathbf{F}^{\chi}=\left(F_{K \pi}^{\chi}(0), \ldots, F_{K \pi}^{\chi}(0), F_{K \eta}^{\chi}(0), \ldots, F_{K \eta}^{\chi}(0)\right)^{T}
$$

[compare to Eq. (30)] and a modified kernel

$$
K_{\mu \nu}\left(s, s^{\prime}\right)=\frac{s}{\pi} \frac{\sigma_{\nu}\left(s^{\prime}\right) T_{\nu \mu}^{*}\left(s^{\prime}\right)}{s^{\prime}} \Theta\left(s^{\prime}-s_{\text {thres } . \nu}\right)
$$

[compare to Eq. (33)]. As Fig. 团 shows, the slope of the chiral NLO expression is not matched any more. Also, the prediction of the form factor at the Callan-Treiman point is wrong. As expected, the uncertainty from the highenergy behavior is significantly larger for the once-subtracted version (shaded areas). Moreover, the difference to the twice-subtracted solution is larger than the uncertainties from the high-energy input.

Finally, we compare to the one-channel version of the form factor. For this, we use the one-channel fit to the phase shift discussed in Sec. IIIA1 and shown in Fig. 2 with the thick dashed (blue) line. The twice-subtracted dispersion relation

$$
F_{K \pi}(s)=F_{K \pi}^{\chi}(0)+\left(F_{K \pi}^{\chi}\right)^{\prime}(0) s+\frac{s^{2}}{\pi} \int_{s_{K \pi}}^{\infty} d s^{\prime} \frac{F_{K \pi}\left(s^{\prime}\right) \sigma_{K \pi}\left(s^{\prime}\right) T_{K \pi, K \pi}^{*}\left(s^{\prime}\right)}{s^{\prime 2}\left(s^{\prime}-s-i \epsilon\right)}
$$

has the Omnès solution (cf., e.g., Ref. [57])

$$
F_{K \pi}(s)=P(s) F_{K \pi}^{\chi}(0) \exp \left[s \frac{\left(F_{K \pi}^{\chi}\right)^{\prime}(0)}{F_{K \pi}^{\chi}(0)}+\frac{s^{2}}{\pi} \int_{s_{K \pi}}^{\infty} \frac{d s^{\prime}}{s^{\prime 2}} \frac{\delta\left(s^{\prime}\right)}{s^{\prime}-s}\right]
$$



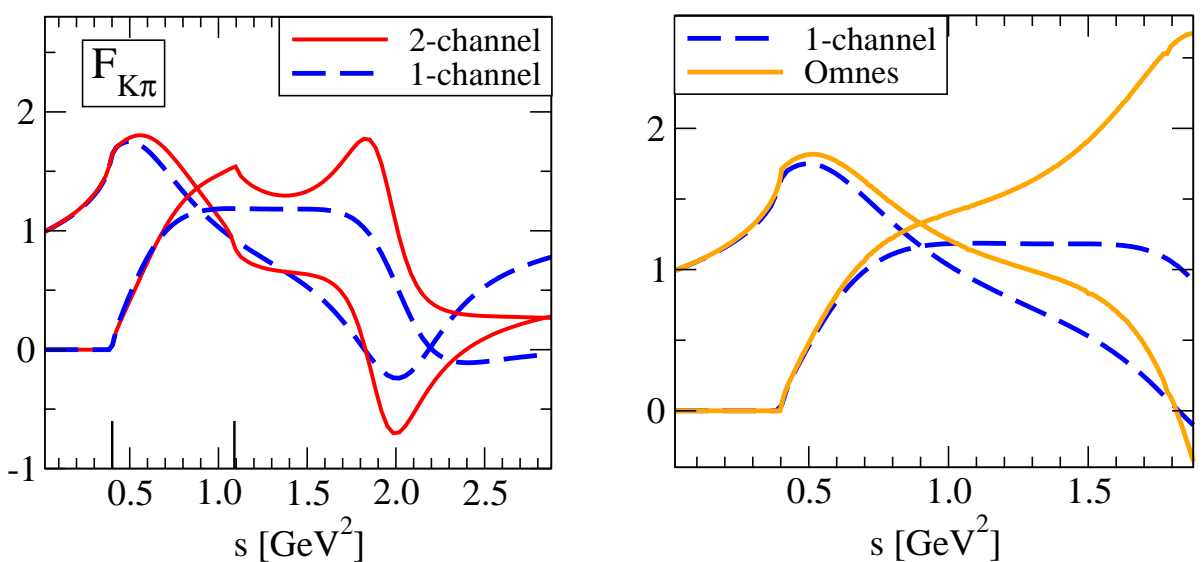

FIG. 5: Left: One-channel $K \pi$ form factor (dashed blue lines) compared to the two-channel solution of Fig. 3 (solid red lines). Right: Direct inversion of Eq. (38) (dashed blue lines) compared to the Omnès solution according to Eq. (39). Both solutions are matched to ChPT at the origin and differ from each other by a real polynomial in $s$ of degree 2 and higher, as must be.

with a polynomial ambiguity $P(s)=1+a_{2} s^{2}+a_{3} s^{3}+\ldots$ (note that $\sigma T^{*}=\sin \delta \exp (-i \delta)$ ).

Using the numerical methods of Sec. IIIA3, Eq. (38) can be directly inverted. To the left in Fig. (5, the corresponding result (dashed blue lines) is compared to the two-channel solution (solid red lines). While the one-channel and the two-channel solutions are qualitatively similar, at higher energies there are differences. In particular, the two-channel solution never becomes zero, while the one-channel solution has a zero around $s=2.2 \mathrm{GeV}^{2}$. In Fig. 5 to the right, the one-channel solution is compared to the Omnès solution of Eq. (39) with $P(s)=1$. The results start to differ already at quite low energies. The Omnès solution cannot become zero while the direct inversion of Eq. (38) exhibits a zero. As we have checked, the two solutions indeed differ by a real polynomial $P(s)$ with a zero, of the form quoted below Eq. (39). The role of zeroes in form factors has extensively been discussed in Ref. [76]. In summary, we observe that the two-channel solution becomes small but stays non-zero, the one-channel solution has a zero, and the Omnès solution cannot have a zero. Experimental data can clarify whether a zero is present or not.

\section{B. Scalar $\pi \pi$ and $K \bar{K}$ form factors}

In terms of the isoscalar $S$-wave states

$$
\begin{aligned}
|\pi \pi\rangle_{\mathrm{I}=0} & =\frac{1}{\sqrt{3}}\left|\pi^{+} \pi^{-}\right\rangle+\frac{1}{\sqrt{6}}\left|\pi^{0} \pi^{0}\right\rangle, \\
|K \bar{K}\rangle_{\mathrm{I}=0} & =\frac{1}{\sqrt{2}}\left|K^{+} K^{-}\right\rangle+\frac{1}{\sqrt{2}}\left|K^{0} \bar{K}^{0}\right\rangle,
\end{aligned}
$$

the scalar form factors for the $\pi$ and $K$ mesons are defined as

$$
\begin{aligned}
& \sqrt{2} B_{0} F_{1}^{n / s}(s)=\langle 0|\bar{n} n / \bar{s} s| \pi \pi\rangle_{\mathrm{I}=0}, \\
& \sqrt{2} B_{0} F_{2}^{n / s}(s)=\langle 0|\bar{n} n / \bar{s} s| K \bar{K}\rangle_{\mathrm{I}=0},
\end{aligned}
$$


where $\bar{n} n=(\bar{u} u+\bar{d} d) / \sqrt{2}$ denotes the non-strange scalar current, and the notation $(\pi=1, K=2)$ has been introduced for simplicity. Expressions have already been derived in CHPT up to next-to-leading order [45, 70, 72, 77]:

$$
\begin{aligned}
F_{1}^{n}(s)= & \sqrt{\frac{3}{2}}\left[1+\mu_{\pi}-\frac{\mu_{\eta}}{3}+\frac{16 m_{\pi}^{2}}{f^{2}}\left(2 L_{8}^{r}-L_{5}^{r}\right)+8\left(2 L_{6}^{r}-L_{4}^{r}\right) \frac{2 m_{K}^{2}+3 m_{\pi}^{2}}{f^{2}}+\frac{8 s}{f^{2}} L_{4}^{r}+\frac{4 s}{f^{2}} L_{5}^{r}\right. \\
& \left.+\left(\frac{2 s-m_{\pi}^{2}}{2 f^{2}}\right) J_{\pi \pi}^{r}(s)+\frac{s}{4 f^{2}} J_{K K}^{r}(s)+\frac{m_{\pi}^{2}}{18 f^{2}} J_{\eta \eta}^{r}(s)\right], \\
F_{1}^{s}(s)= & \frac{\sqrt{3}}{2}\left[\frac{16 m_{\pi}^{2}}{f^{2}}\left(2 L_{6}^{r}-L_{4}^{r}\right)+\frac{8 s}{f^{2}} L_{4}^{r}+\frac{s}{2 f^{2}} J_{K K}^{r}(s)+\frac{2}{9} \frac{m_{\pi}^{2}}{f^{2}} J_{\eta \eta}^{r}(s)\right], \\
F_{2}^{n}(s)= & \frac{1}{\sqrt{2}}\left[1+\frac{8 L_{4}^{r}}{f^{2}}\left(2 s-m_{\pi}^{2}-6 m_{K}^{2}\right)+\frac{4 L_{5}^{r}}{f^{2}}\left(s-4 m_{K}^{2}\right)+\frac{16 L_{6}^{r}}{f^{2}}\left(6 m_{K}^{2}+m_{\pi}^{2}\right)+\frac{32 L_{8}^{r}}{f^{2}} m_{K}^{2}+\frac{2}{3} \mu_{\eta}\right. \\
& \left.+\left(\frac{9 s-8 m_{K}^{2}}{36 f^{2}}\right) J_{\eta \eta}^{r}(s)+\frac{3 s}{4 f^{2}} J_{K K}^{r}(s)+\frac{3 s}{4 f^{2}} J_{\pi \pi}^{r}(s)\right], \\
F_{2}^{s}(s)= & +\frac{8 L_{4}^{r}}{f^{2}}\left(s-m_{\pi}^{2}-4 m_{K}^{2}\right)+\frac{4 L_{5}^{r}}{f^{2}}\left(s-4 m_{K}^{2}\right)+\frac{16 L_{6}^{r}}{f^{2}}\left(4 m_{K}^{2}+m_{\pi}^{2}\right)+\frac{32 L_{8}^{r}}{f^{2}} m_{K}^{2}+\frac{2}{3} \mu_{\eta} \\
& +\left(\frac{9 s-8 m_{K}^{2}}{18 f^{2}}\right) J_{\eta \eta}^{r}(s)+\frac{3 s}{4 f^{2}} J_{K K}^{r}(s) .
\end{aligned}
$$

Imposing the unitarity constraints, the scalar form factor can be expressed in terms of the algebraic coupled-channel equation

$$
\begin{aligned}
F(s) & =[I+K(s) g(s)]^{-1} R(s) \\
& =[I-K(s) g(s)] R(s)+\mathcal{O}\left(p^{6}\right),
\end{aligned}
$$

where $R(s)$ has no right-hand cut and in the second line, the equation has been expanded up to NLO in the chiral expansion. $K(s)$ is the $S$-wave projected kernel of meson-meson scattering amplitudes that can be derived from the leading-order chiral Lagrangian:

$$
K_{11}=\frac{2 s-m_{\pi}^{2}}{2 f^{2}}, \quad K_{12}=K_{21}=\frac{\sqrt{3} s}{4 f^{2}}, \quad K_{22}=\frac{3 s}{4 f^{2}} .
$$

The loop integral can be calculated either in the cutoff-regularization scheme with $q_{\max } \sim 1 \mathrm{GeV}$ being the cutoff [cf. Erratum of Ref. 61] for an explicit expression] or in dimensional regularization with the modified $\overline{\mathrm{MS}}$ subtraction scheme. In the latter scheme, the meson loop function $g_{i}(s)$ is given by

$$
\begin{aligned}
J_{i i}^{r}(s) & \equiv \frac{1}{16 \pi^{2}}\left[1-\log \left(\frac{m_{i}^{2}}{\mu^{2}}\right)-\sigma_{i}(s) \log \left(\frac{\sigma_{i}(s)+1}{\sigma_{i}(s)-1}\right)\right] \\
& =-g_{i}(s) .
\end{aligned}
$$

with $\sigma_{i}(s)=\sqrt{1-4 m_{i}^{2} / s}$. The matching between these two renormalization is given in Eq. (22) with $q_{\max }=\Lambda$. 

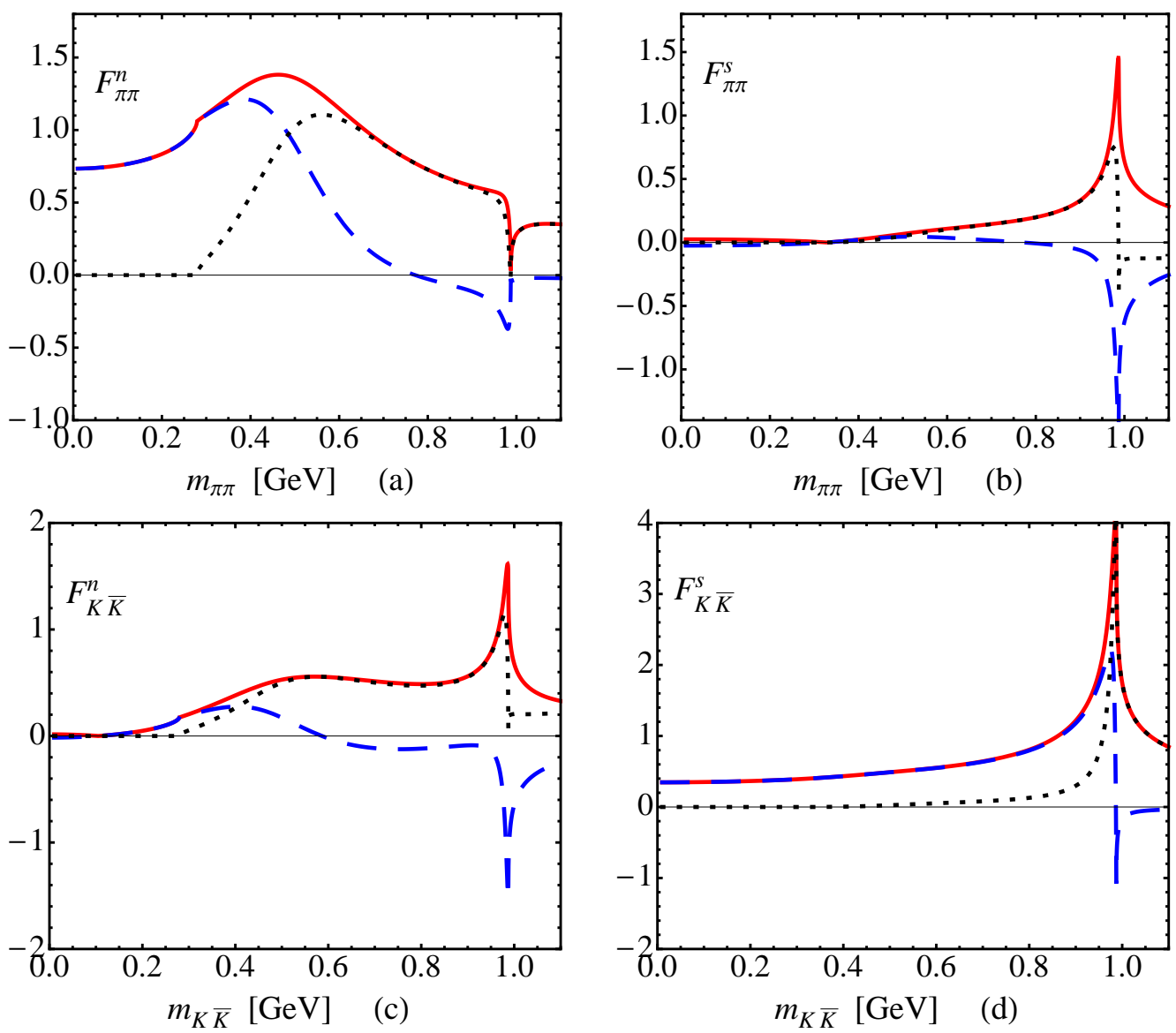

FIG. 6: The non-strange and strange $\pi \pi$ and $K \bar{K}$ scalar form factors obtained in the unitarized chiral perturbation theory. The modulus, real part and imaginary part are shown in solid, dashed and dotted curves.

The expressions for the $R^{i}$ are obtained by matching the unitarization and chiral perturbation theory [49, 78]:

$$
\begin{aligned}
R_{1}^{n}(s)= & \sqrt{\frac{3}{2}}\left\{1+\mu_{\pi}-\frac{\mu_{\eta}}{3}+\frac{16 m_{\pi}^{2}}{f^{2}}\left(2 L_{8}^{r}-L_{5}^{r}\right)+8\left(2 L_{6}^{r}-L_{4}^{r}\right) \frac{2 m_{K}^{2}+3 m_{\pi}^{2}}{f^{2}}+\frac{8 s}{f^{2}} L_{4}^{r}+\frac{4 s}{f^{2}} L_{5}^{r}\right. \\
& \left.-\frac{m_{\pi}^{2}}{288 \pi^{2} f^{2}}\left[1+\log \left(\frac{m_{\eta}^{2}}{\mu^{2}}\right)\right]\right\}, \\
R_{1}^{s}(s)= & \frac{\sqrt{3}}{2}\left\{\frac{16 m_{\pi}^{2}}{f^{2}}\left(2 L_{6}^{r}-L_{4}^{r}\right)+\frac{1}{3} \frac{8 s}{f^{2}} L_{4}^{r}-\frac{m_{\pi}^{2}}{72 \pi^{2} f^{2}}\left[1+\log \left(\frac{m_{\eta}^{2}}{\mu^{2}}\right)\right]\right\}, \\
R_{2}^{n}(s)= & \frac{1}{\sqrt{2}}\left\{1+\frac{8 L_{4}^{r}}{f^{2}}\left(2 s-6 m_{K}^{2}-m_{\pi}^{2}\right)+\frac{4 L_{5}^{r}}{f^{2}}\left(s-4 m_{K}^{2}\right)+\frac{16 L_{6}^{r}}{f^{2}}\left(6 m_{K}^{2}+m_{\pi}^{2}\right)+\frac{32 L_{8}^{r}}{f^{2}} m_{K}^{2}+\frac{2}{3} \mu_{\eta}\right. \\
& \left.+\frac{m_{K}^{2}}{72 \pi^{2} f^{2}}\left[1+\log \left(\frac{m_{\eta}^{2}}{\mu^{2}}\right)\right]\right\}, \\
R_{2}^{s}(s)= & 1+\frac{8 L_{4}^{r}}{f^{2}}\left(s-4 m_{K}^{2}-m_{\pi}^{2}\right)+\frac{4 L_{5}^{r}}{f^{2}}\left(s-4 m_{K}^{2}\right)+\frac{16 L_{6}^{r}}{f^{2}}\left(4 m_{K}^{2}+m_{\pi}^{2}\right)+\frac{32 L_{8}^{r}}{f^{2}} m_{K}^{2}+\frac{2}{3} \mu_{\eta} \\
& +\frac{m_{K}^{2}}{36 \pi^{2} f^{2}}\left[1+\log \left(\frac{m_{\eta}^{2}}{\mu^{2}}\right)\right],
\end{aligned}
$$

where the factor $1 / 3$ in $R_{1}^{s}(s)$ is missing in Ref. [49].

With the above formulae and the fitted results for the low-energy constants $L_{i}^{r}$ in Ref. [49] (evolved from $M_{\rho}$ to 

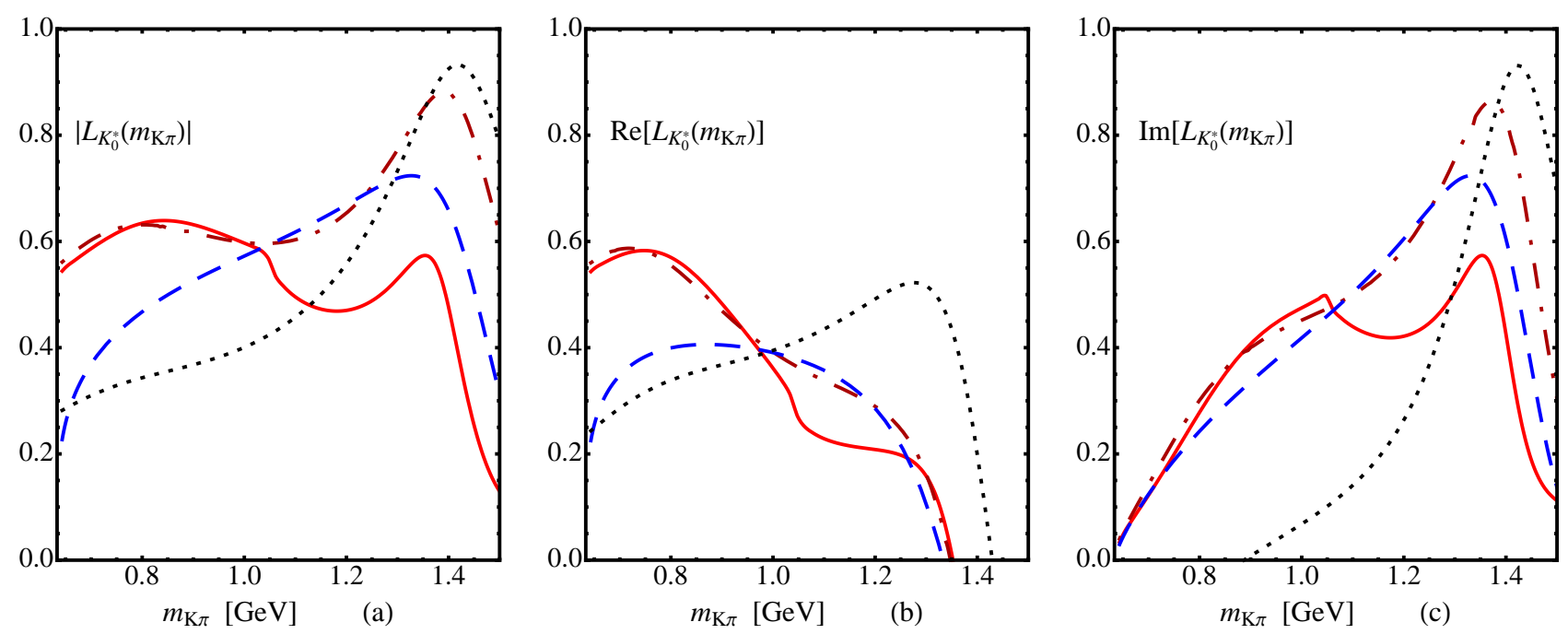

FIG. 7: S-wave $K \pi$ line shapes. The modulus, real part, and imaginary part are given in panel (a), (b), and (c), respectively. Solid (red) lines: lineshape given by Eq. (9) with the Muskhelishvili-Omnès solution of Eq. (24) for the form factor. Dash-dotted (dark red) lines: Omnès solution of Eq. (39). Dotted lines: LASS lineshape of Eq. (55); dashed (blue) lines: parameterization of Eq. (58).

the scale $\mu=2 q_{\max } / \sqrt{e}$ ), we show the non-strange and strange $\pi \pi$ and $K \bar{K}$ form factors in Fig. 6. The modulus, real part and imaginary part are shown as solid, dashed and dotted curves. As the figure shows, the chiral unitary ansatz predicts a form factor $F_{\pi \pi}^{n}$ with a zero close to the $\bar{K} K$ threshold. This feature has been extensively discussed in Ref. [76].

\section{RESULTS AND DISCUSSIONS}

In the narrow-width limit, the integration over the $K \pi$ invariant mass leads to the normalization of the $K^{*}\left[K^{*} \equiv\right.$ $\left.K^{*}(892)\right]$ line shape:

$$
\int d m_{K \pi}^{2}\left|L_{K^{*}}\left(m_{K \pi}^{2}\right)\right|^{2}=\mathcal{B}\left(\bar{K}^{* 0} \rightarrow K^{-} \pi^{+}\right) \simeq \frac{2}{3}
$$

with $\mathcal{B}$ being the branching ratio. Considering the momentum dependence of the $K^{*}$ decay, we have the running width as

$$
\Gamma_{K^{*}}\left(m_{K \pi}^{2}\right)=\Gamma_{K^{*}}^{0}\left(\frac{|\vec{q}|}{\left|\overrightarrow{q_{0}}\right|}\right)^{3} \frac{m_{K^{*}}}{m_{K \pi}} \frac{1+\left(R\left|\vec{q}_{0}\right|\right)^{2}}{1+(R|\vec{q}|)^{2}}
$$

and the Blatt-Weisskopf parameter $R=(2.1 \pm 0.5 \pm 0.5) \mathrm{GeV}^{-1}$ [21]. It is plausible to assume the same form for the $\phi$ meson except that the mass, total decay width and the branching ratios into $K^{+} K^{-}$are replaced correspondingly.

We start with the numerical discussion on the $K^{-} \pi^{+}$and $K^{+} K^{-}$line-shapes. In the case of $K^{-} \pi^{+}$, we compare with the data-inspired LASS parametrization [29, 79]

$$
L_{K_{0}^{*}}^{\mathrm{LASS}}\left(m_{K \pi}\right)=\mathcal{N}_{\mathrm{LASS}}\left(\frac{m_{K \pi}}{|\vec{q}|\left(\cot \delta_{B}-i\right)}+e^{2 i \delta_{B}} \frac{m_{K_{0}^{*}}^{2} \Gamma_{K_{0}^{*}}^{0} /\left|\vec{q}_{0}\right|}{m_{K_{0}^{*}}^{2}-m_{K \pi}^{2}-i m_{K_{0}^{*}} \Gamma_{K_{0}^{*}}\left(m_{K \pi}^{2}\right)}\right),
$$

with $\vec{q}$ and $\vec{q}_{0}$ being three momentum of kaon/pion at $m_{K \pi}$ and $m_{K_{0}^{*}}$, and the parameters [80]

$$
\cot \delta_{B}=\frac{1}{a|\vec{q}|}+\frac{1}{2} r|\vec{q}|, \quad a=1.94 \mathrm{GeV}^{-1}, \quad r=1.76 \mathrm{GeV}^{-1}
$$


The running decay width in Eq. (55) is

$$
\Gamma_{K_{0}^{*}}\left(m_{K \pi}^{2}\right)=\Gamma_{K_{0}^{*}}^{0} \frac{|\vec{q}|}{\left|\vec{q}_{0}\right|} \frac{m_{K_{0}^{*}}}{m_{K \pi}} .
$$

The constant $\mathcal{N}_{\text {LASS }}$ in Eq. (55) can be chosen such that the $K_{0}^{*}(1430)$ term has the same normalization as the ordinary Breit-Wigner formula. In the study of S-wave effects in $B \rightarrow K^{*} l^{+} l^{-}$, the authors of Ref. [18] have suggested a phenomenological parametrization:

$$
L_{K_{0}^{*}}^{\mathrm{BT}}=\mathcal{N}_{\mathrm{BT}}\left[\frac{g_{\kappa}}{m_{K \pi}^{2}-\left(m_{\kappa}-i \Gamma_{\kappa} / 2\right)^{2}}-\frac{1}{m_{K \pi}^{2}-\left(m_{K_{0}^{*}(1430)}-i \Gamma_{K_{0}^{*}(1430)} / 2\right)^{2}}\right],
$$

with the coupling constant chosen by hand $g_{\kappa} \sim 0.2$ and $\mathcal{N}_{B T}$ as the normalization constant.

In Fig 7 , we compare the three parametrizations defined in Eq. (9), Eq. (55), and Eq. (58) corresponding to the solid, dotted and dashed curves with the result of Eq. (9) given by the Muskhelishvili-Omnès solution according to Eq. (24) and shown in Fig. 3. Dash-dotted lines are the Omnès solution of Eq. (39). In the experimental study of $B \rightarrow$ $J / \psi K \pi$ [80], the Babar collaboration found in their Fig.11 that the LASS parametrization for S-wave contribution will undershoot the experimental data in the low $K \pi$ invariant mass region, in particular around $m_{K \pi} \sim 0.7 \mathrm{GeV}$. As Fig. 7 shows for $\left|L_{K_{0}^{*}}\left(m_{K \pi}\right)\right|$, the results derived in this study can indeed improve this underprediction at low invariant masses. Both the Muskhelishvili-Omnès and the Omnès solution have more strength at low invariant masses than the LASS parametrization. In both results of this study, a double hump structure from the $\kappa$ and the $K_{0}^{*}(1430)$ is observed. In the two-channel solution (solid red line), the flux into the $K \eta$ channel significantly reduces the strength at the $K_{0}^{*}(1430)$ position, which is not the case for the one-channel (elastic) Omnès solution (dash-dotted lines). As discussed before, the latter cannot have a zero and thus might overpredict the strength at the $K_{0}^{*}(1430)$ position.

We will also study the process $B_{s} \rightarrow \phi\left(\rightarrow K^{+} K^{-}\right) l^{+} l^{-}$, where the $K \bar{K}$ state is close to threshold and the S-wave mass distribution is well-described by the Flatté model [81]

$$
L_{f_{0}(980)}\left(m_{K \bar{K}}\right)=\sqrt{\frac{m_{f_{0}} \Gamma_{f_{0} \rightarrow K^{+} K^{-}}}{\pi}} \frac{1}{m_{K \bar{K}}^{2}-m_{f_{0}}^{2}+i m_{K \bar{K}}\left(g_{1} \rho_{\pi \pi}+g_{2} \rho_{K \bar{K}}\right)},
$$

with $\rho_{\pi \pi / K \bar{K}}$ being the Lorentz-invariant phase space, $\rho_{\pi \pi / K \bar{K}}=2|\vec{q}| / m_{K \bar{K}}=\sqrt{1-4 m_{\pi / K}^{2} / m_{K \bar{K}}^{2}}$. The involved couplings have been measured from $J / \psi$ decays by the BES collaboration [82]

$$
m_{f_{0}} \Gamma_{f_{0} \rightarrow K^{+} K^{-}}=\frac{1}{2} g_{2} \rho_{K \bar{K}}, \quad g_{1}=(165 \pm 10 \pm 15) \mathrm{MeV}, \quad g_{2} / g_{1}=4.21 \pm 0.25 \pm 0.21 .
$$

To quantitatively demonstrate the size of S-wave contributions, we study the total differential decay width $d \Gamma / d q^{2}$ (integrated over $m_{K \pi}$ ), the S-wave fraction and the forward-backward asymmetry distribution for the charged kaon, $d A_{F B}^{K} / d q^{2}$ :

$$
\begin{aligned}
& \frac{d \Gamma}{d q^{2}} \equiv \int_{\left(m_{K^{*}}-\delta m\right)^{2}}^{\left(m_{K^{*}}+\delta m\right)^{2}} d m_{K \pi}^{2} \frac{d^{2} \Gamma}{d q^{2} d m_{K \pi}^{2}}, \\
& \frac{d \Gamma_{S}}{d q^{2}} \equiv \int_{\left(m_{K^{*}}-\delta m\right)^{2}}^{\left(m_{K^{*}}+\delta m\right)^{2}} d m_{K \pi}^{2} \frac{d^{2} \Gamma_{S}}{d q^{2} d m_{K \pi}^{2}}=\int_{\left(m_{K^{*}}-\delta m\right)^{2}}^{\left(m_{K^{*}}+\delta m\right)^{2}} d m_{K \pi}^{2}\left[\left|A_{L 0}\right|^{2}+\left|A_{R 0}\right|^{2}\right], \\
& \frac{d A_{F B}^{K}}{d q^{2}} \equiv \int_{\left(m_{K^{*}}-\delta m\right)^{2}}^{\left(m_{K^{*}}+\delta m\right)^{2}} d m_{K \pi}^{2} \frac{d^{2} A_{F B}^{K}}{d q^{2} d m_{K \pi}^{2}}, \quad \frac{\overline{d A_{F B}^{K}}}{d q^{2}} \equiv \frac{\frac{d A_{F B}^{K}}{d q^{2}}}{\frac{d \Gamma}{d q^{2}}},
\end{aligned}
$$

with $m_{K^{*}} \equiv m_{K^{*}(892)}$.

With the choice of $\delta_{m}=100 \mathrm{MeV}$ (the default choice adopted by the LHCb collaboration [4]), we show our results for $d \Gamma / d q^{2}$ in panel (a) of Fig. 8 , where the dashed, dotted and solid curves denote the S-wave, P-wave and total 

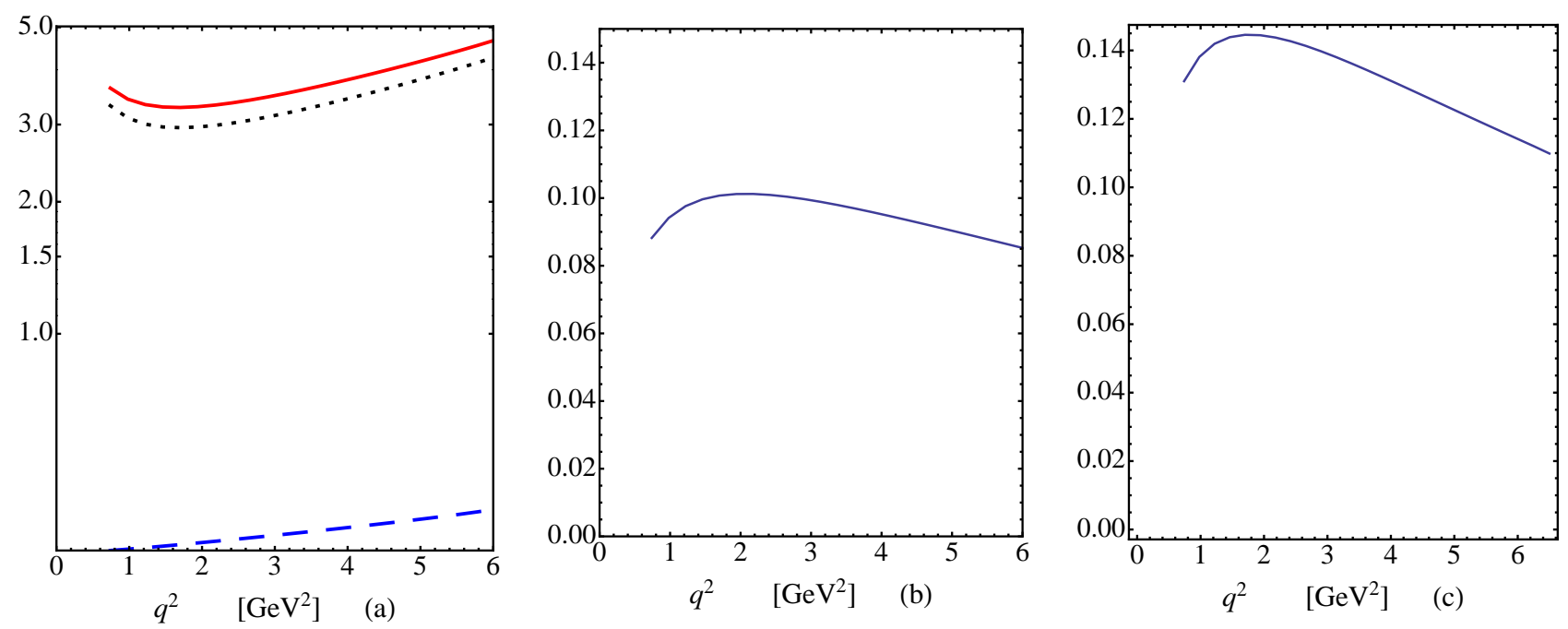

FIG. 8: The S-wave contribution and its interference with $\mathrm{P}$-wave to the differential decay width in $\bar{B}^{0} \rightarrow K^{-} \pi^{+} l^{+} l^{-}$. In panel (a), the dashed, dotted and solid curves denote the S-wave, $\mathrm{P}$-wave and total contributions $d \Gamma / d q^{2}$ in $\mathrm{units}^{\mathrm{i}}$ of $10^{-8} \mathrm{GeV}^{-2}$. Panel (b) shows the S-wave fraction $d \Gamma_{S} / d q^{2}$. We also show the forward-back asymmetry $d A_{F B}^{K} / d q^{2}$ for the charged kaon in panel (c).
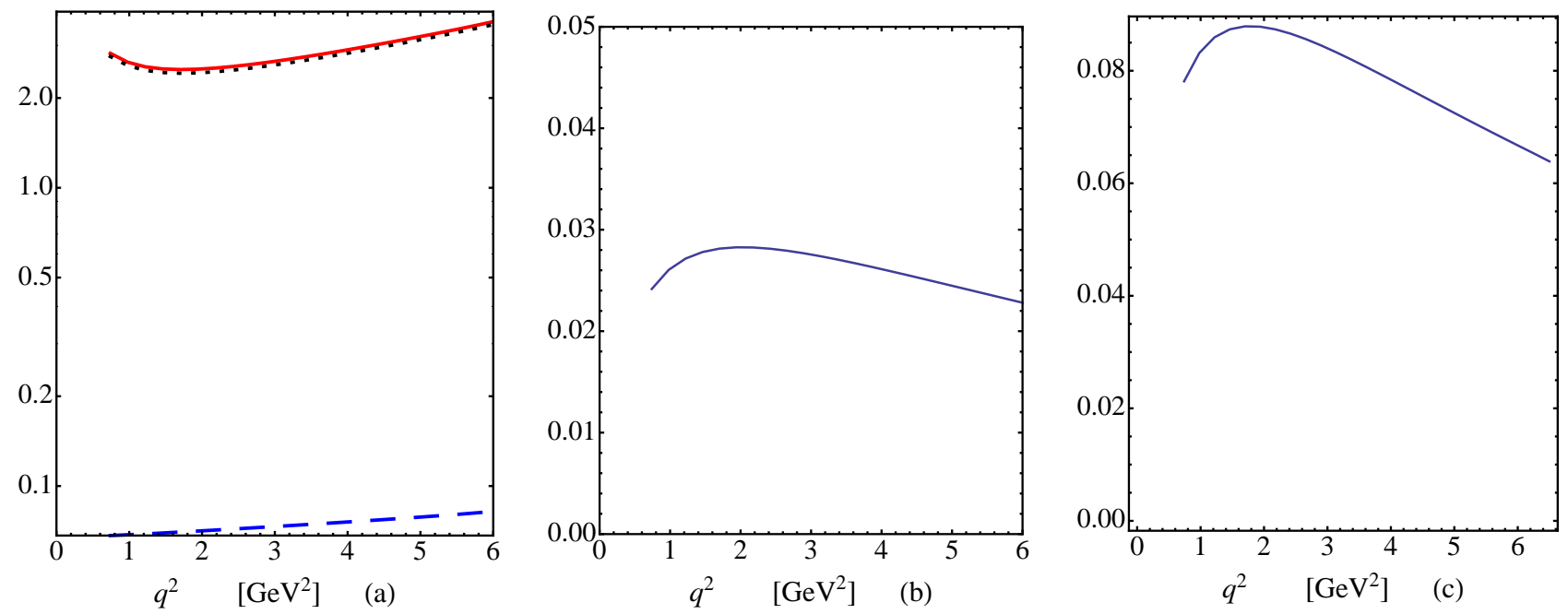

FIG. 9: Same as Fig. 8 but for $\bar{B}_{s}^{0} \rightarrow K^{+} K^{-} l^{+} l^{-}$.

contributions respectively. The panel (b) and (c) correspond to the S-wave fraction and and the forward-backward asymmetry. In the case of $B_{s} \rightarrow \phi l^{+} l^{-}$the bin size is chosen as $\delta_{m}=20 \mathrm{MeV}$ and the corresponding results are shown in Fig. 9. From these figures, we find that the S-wave contribution can reach $10 \%$ in $\bar{B}^{0} \rightarrow K^{-} \pi^{+} l^{+} l^{-}$, while it is about $5 \%$ in $B_{s} \rightarrow K^{+} K^{-} l^{+} l^{-}$. It is necessary to stress that there is a sign ambiguity in the forward-backward asymmetry $d A_{F B}^{K} / d q^{2}$ from the use of Watson theorem. However, the magnitude shown in panel (c) is sizable and thus measurable in future. Since this quantity $d A_{F B}^{K} / d q^{2}$ arises from the S-wave and P-wave interference, it can be used to constrain the $\mathrm{S}$-wave meson-meson scattering when precise data is available in future.

Based on the data sample of $1 \mathrm{fb}^{-1}$, the LHCb collaboration has set an upper limit for the integrated S-wave fraction 

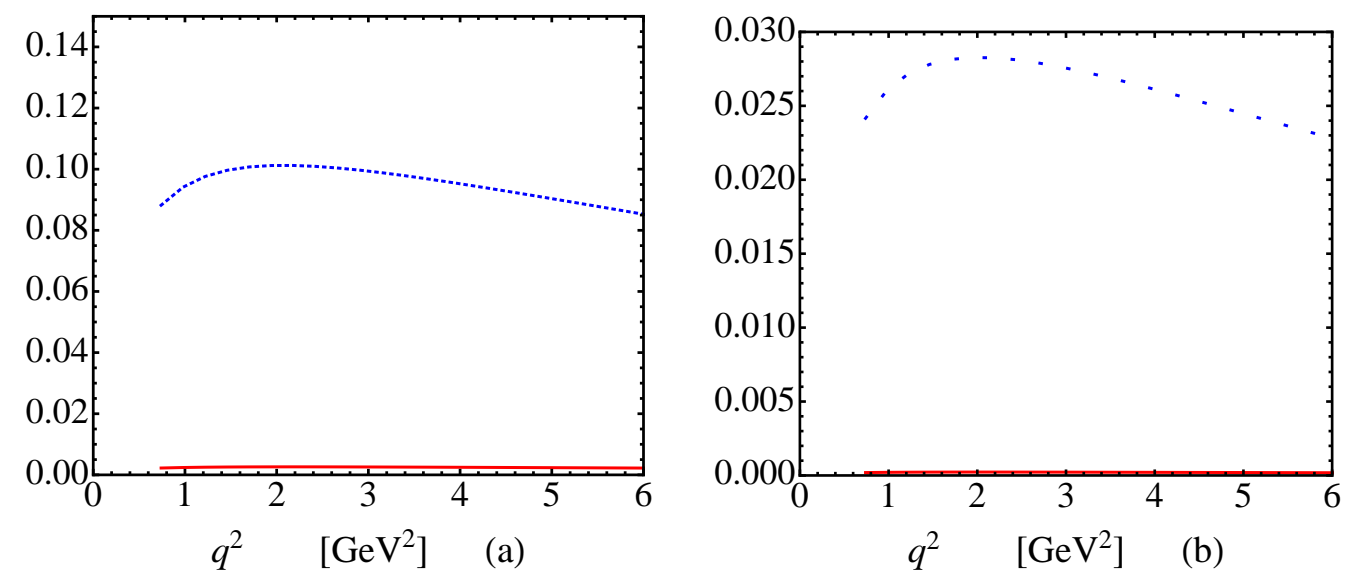

FIG. 10: Fraction of S-wave contributions shown in the dotted (blue) curves in $\bar{B}^{0} \rightarrow K^{-} \pi^{+} l^{+} l^{-}$(left panel) and $\bar{B}_{s} \rightarrow$ $K^{+} K^{-} l^{+} l^{-}$(right panel). After using the kinematical subtraction in Eq. (63), the S-wave contributions are reduced to less than $1 \%$.

$F_{S}<0.07$ at $68 \% \mathrm{CL}$, in the range $1 \mathrm{GeV}^{2}<q^{2}<6 \mathrm{GeV}^{2}[4]$ and

$$
F_{S}=0.04 \pm 0.04
$$

Our estimate is slightly larger than this but still consistent with the data when errors are taken into account.

To minimize the S-wave contributions and project out the $\mathrm{P}$-wave, we propose to study the subtracted differential decay width:

$$
\begin{aligned}
& \frac{d \Gamma^{\prime}}{d q^{2}} \equiv\left[2 \int_{\left(m_{K^{*}}-\delta m_{2}\right)^{2}}^{\left(m_{K^{*}}+\delta m_{2}\right)^{2}}-\int_{\left(m_{K^{*}}-\delta m_{1}\right)^{2}}^{\left(m_{\left.K^{*}+\delta m_{1}\right)^{2}}\right.}\right] d m_{K \pi}^{2} \frac{d^{2} \Gamma}{d q^{2} d m_{K \pi}^{2}} \\
& =\left[\int_{\left(m_{K^{*}}-\delta m_{2}\right)^{2}}^{\left(m_{K^{*}}+\delta m_{2}\right)^{2}}-\int_{\left(m_{K^{*}}-\delta m_{1}\right)^{2}}^{\left(m_{K^{*}}-\delta m_{2}\right)^{2}}-\int_{\left(m_{K^{*}}+\delta m_{2}\right)^{2}}^{\left(m_{K^{*}}+\delta m_{1}\right)^{2}}\right] d m_{K \pi}^{2} \frac{d^{2} \Gamma}{d q^{2} d m_{K \pi}^{2}},
\end{aligned}
$$

with $\delta m_{1}=2 \delta m_{2}$. The physical interpretation is to slice the $K \pi$ invariant mass distribution into 4 bins around $m_{K \pi} \sim m_{K^{*}(892)}$ with $\delta m_{2}$ being the size of the bins. We select the events in the central two bins and subtract those from the other two bins. For illustration we use $\delta_{m_{1}}=2 \delta_{m_{2}}=100 \mathrm{MeV}$ for $B \rightarrow K^{-} \pi^{+} l^{+} l^{-}$and $\delta_{m_{1}}=2 \delta_{m_{2}}=20$ $\mathrm{MeV}$ for $B_{s} \rightarrow K^{+} K^{-} l^{+} l^{-3}$. From the results in Fig. 10, we can see the S-wave contributions have been reduced to less than $1 \%$ for both $B \rightarrow K^{-} \pi^{+} l^{+} l^{-}$and $B_{s} \rightarrow K^{+} K^{-} l^{+} l^{-}$. The disadvantage is the demand of larger statistics.

Before closing this section, several remarks are given in order.

- We have considered the invariant mass distribution for the light meson pair using chiral perturbation theory and dispersion relations, but used the heavy-to-light form factors calculated in the resonance approximation.

- Our analysis will be improved by a direct calculation of the matrix element $\left\langle(K \pi)_{0}|\bar{s} \Gamma b| \bar{B}\right\rangle$ and also hardscattering QCD corrections. This can be achieved in the factorization approach by merging the perturbative nature of $\mathrm{B}$ decays and the chiral perturbation description of $K \pi$. For future use, we parametrize these matrix

3 The CDF collaboration has used the choice of $\delta_{m}=50 \mathrm{MeV}$ and $\delta_{m}=10 \mathrm{MeV}$ for $K^{+} \pi^{-}$and $K^{+} K^{-}$states in the analysis of $B \rightarrow K^{*} l^{+} l^{-}$and $B_{s} \rightarrow \phi l^{+} l^{-}$decays [3], while LHCb adopted $\delta_{m}=100 \mathrm{MeV}$ for $K \pi$ [4], and $\delta_{m}=12 \mathrm{MeV}$ for $K^{+} K^{-}$[83]. 
elements in the following

$$
\begin{aligned}
\left\langle(K \pi)_{0}\left(p_{K \pi}\right)\left|\bar{s} \gamma_{\mu} \gamma_{5} b\right| \bar{B}\left(p_{B}\right)\right\rangle & =-i \frac{1}{m_{K \pi}}\left\{\left[P_{\mu}-\frac{m_{B}^{2}-m_{K \pi}^{2}}{q^{2}} q_{\mu}\right] \mathcal{F}_{1}\left(m_{K \pi}^{2}, q^{2}\right)+\frac{m_{B}^{2}-m_{K \pi}^{2}}{q^{2}} q_{\mu} \mathcal{F}_{0}\left(m_{K \pi}^{2}, q^{2}\right)\right\}, \\
\left\langle(K \pi)_{0}\left(p_{K \pi}\right)\left|\bar{s} \sigma_{\mu \nu} q^{\nu} \gamma_{5} b\right| \bar{B}\left(p_{B}\right)\right\rangle & =\frac{\mathcal{F}_{T}\left(m_{K \pi}^{2}, q^{2}\right)}{m_{K \pi}\left(m_{B}+m_{K \pi}\right)}\left[\left(m_{B}^{2}-m_{K \pi}^{2}\right) q_{\mu}-q^{2} P_{\mu}\right],
\end{aligned}
$$

where $\mathcal{F}_{1,0, T}\left(m_{K \pi}, q^{2}\right)$ are the "generalized" form factors and an additional dependence on kaon/pion momentum is suppressed here. Further, $P=p_{B}+p_{K \pi}$ and $q=p_{B}-p_{K \pi}$. Fortunately, the heavy quark and large recoil symmetries are valid in the small $q^{2}$ region, and thus we have the large-recoil symmetry at the leading-order in $\alpha_{s}$ and $1 / m_{b}[84]$ :

$$
\mathcal{F}_{1}\left(m_{K \pi}, q^{2}\right)=\frac{m_{B}^{2}-m_{K \pi}^{2}}{m_{B}^{2}-m_{K \pi}^{2}-q^{2}} \mathcal{F}_{0}\left(m_{K \pi}, q^{2}\right)=\frac{m_{B}}{m_{B}+m_{K \pi}} \mathcal{F}_{T}\left(m_{K \pi}, q^{2}\right) \equiv \xi\left(m_{K \pi}, q^{2}\right) .
$$

- The calculation of generalized form factors $\mathcal{F}_{1,0, T}\left(m_{K \pi}, q^{2}\right)$ and hard-scattering QCD corrections will remove the necessity for the use of Watson theorem, but requires the knowledge of the $K \pi$ generalized light-cone distribution amplitudes, defined in the following form for the $K \pi$ system with spin $J$ :

$$
\left\langle(K \pi)_{J}|\bar{s}(z) \Gamma q(-z)| 0\right\rangle .
$$

Here $\Gamma$ denotes a generic Dirac matrix. It is interesting to notice these distribution amplitudes are normalized to scalar form factors which were already discussed in the last section. In the $\pi \pi$ case, the leading-twist distribution amplitude has been derived using CHPT [85], while to the best of our knowledge the sub-leading twist distribution amplitudes are not yet available from first-principles in the literature.

- Our analysis can be generalized to the $b \rightarrow u l \bar{\nu}$ processes like $B^{-} \rightarrow \pi^{+} \pi^{-} l \bar{\nu}$ and $\bar{B}_{s}^{0} \rightarrow K^{0} \pi^{+} l \bar{\nu}$, which are of great interest for the determination of CKM matrix element $\left|V_{u b}\right|$.

- Similarly, if two final mesons are moving collinear (with small invariant mass) in three-body $B$ decays like $B \rightarrow K K \bar{K}, K \pi \pi, K \bar{K} \pi, \pi \pi \pi$, the decay matrix element will be factorized at leading order in $1 / m_{b}$. Depending on the topology of the two-meson system, decay amplitudes are expressed in terms of a product of the generalized form factors for the recoiling two-meson system as defined in Eq. (64) and the light-cone distribution amplitude for the emitted meson, or the heavy-to-light form factors for the recoil meson and generalized distribution amplitude for the emitted two-meson system. See for example Refs. [86 88] for some discussions along this line.

\section{CONCLUSION}

Our understanding of standard model and CP violation benefits a lot from heavy flavor physics, and thereby considerable amount of effort has been made in recent years. Since the momentum transfer in $B$ decays is large, one can use the factorization scheme to separate the short-distance and the long-distance physics. The calculation of the short-distance part is based on perturbation theory and operator product expansion in QCD, and has reached a high precision. The long-distance matrix element is usually challenged by our knowledge of the S-wave. In this work, we point out the S-wave contribution can be controlled using chiral perturbation theory, which offers a systematic way for the study of the S-wave in $B$ semi-leptonic and non-leptonic decays. Still, direct measurements have to be made to disentangle remaining polynomial ambiguities.

In this work, we considered the example $B \rightarrow K \pi l^{+} l^{-}$and identified the matrix element $\langle K \pi|\bar{s} \Gamma b| \bar{B}\rangle$ with the S-wave scalar form factor. Using the S-wave $K \pi$ and $K \bar{K}$ form factors from the Muskhelishvili-Omnès solution and unitarized chiral perturbation theory, respectively, we have investigated the S-wave contributions in the decay $\bar{B}^{0} \rightarrow K^{-} \pi^{+} l^{+} l^{-}$ 
with the $K \pi$ invariant mass lying in the vicinity of the mass of the $K^{*}(892)$ and the decay $B_{s} \rightarrow K^{-} K^{+} l^{+} l^{-}$with $m_{K K} \sim m_{\phi}$. We found that differential decay widths are affected by about $10 \%$ in the process of $\bar{B}^{0} \rightarrow K^{-} \pi^{+} l^{+} l^{-}$, which is larger than but still consistent with the LHCb measurements. A forward-backward asymmetry for the charged kaon in the final state arises due to the interference between S-wave and P-wave contributions. The measurement of this asymmetry in the future offers a new way to constrain the variation of the $K \pi$ S-wave phase versus the invariant mass and it should be compared with other experimental determinations.

\section{Acknowledgements}

We are grateful to Feng-Kun Guo, C. Hanhart, Bastian Kubis and J.A. Oller for enlightening discussions. W.W. thanks Ignacio Bediaga and Manoel Robilotta for valuable discussions during the FPCP2013 conference. This work is supported in part by the DFG and the NSFC through funds provided to the Sino-German CRC 110 "Symmetries and the Emergence of Structure in QCD", the "EU I3HP Study of Strongly Interacting Matter" under the Seventh Framework Program of the EU.

\section{Appendix A: $B \rightarrow K_{J}^{*}$ Form factors}

The $B \rightarrow K_{0}^{*}$ form factors are parametrized as

$$
\begin{aligned}
\left\langle K_{0}^{*}\left(P_{2}\right)\left|\bar{s} \gamma_{\mu} \gamma_{5} b\right| \bar{B}\left(P_{B}\right)\right\rangle & =-i\left\{\left[P_{\mu}-\frac{m_{B}^{2}-m_{K_{0}^{*}}^{2}}{q^{2}} q_{\mu}\right] F_{1}\left(q^{2}\right)+\frac{m_{B}^{2}-m_{K_{0}^{*}}^{2}}{q^{2}} q_{\mu} F_{0}\left(q^{2}\right)\right\}, \\
\left\langle K_{0}^{*}\left(P_{2}\right)\left|\bar{s} \sigma_{\mu \nu} q^{\nu} \gamma_{5} b\right| \bar{B}\left(P_{B}\right)\right\rangle & =\left[\left(m_{B}^{2}-m_{K_{0}^{*}}^{2}\right) q_{\mu}-q^{2} P_{\mu}\right] \frac{F_{T}\left(q^{2}\right)}{m_{B}+m_{K_{0}^{*}}},
\end{aligned}
$$

while the $B \rightarrow K_{J}^{*}(J \geqslant 1)$ form factors are defined by 89 91]

$$
\begin{aligned}
\left\langle K_{J}^{*}\left(P_{2}, \epsilon\right)\left|\bar{s} \gamma^{\mu} b\right| \bar{B}\left(P_{B}\right)\right\rangle= & -\frac{2 V\left(q^{2}\right)}{m_{B}+m_{K_{J}^{*}}} \epsilon^{\mu \nu \rho \sigma} \epsilon_{J \nu}^{*} P_{B \rho} P_{2 \sigma}, \\
\left\langle K_{J}^{*}\left(P_{2}, \epsilon\right)\left|\bar{s} \gamma^{\mu} \gamma_{5} b\right| \bar{B}\left(P_{B}\right)\right\rangle= & 2 i m_{K_{J}^{*}} A_{0}\left(q^{2}\right) \frac{\epsilon_{J}^{*} \cdot q}{q^{2}} q^{\mu}+i\left(m_{B}+m_{K_{J}^{*}}\right) A_{1}\left(q^{2}\right)\left[\epsilon_{J \mu}^{*}-\frac{\epsilon_{J}^{*} \cdot q}{q^{2}} q^{\mu}\right] \\
& -i A_{2}\left(q^{2}\right) \frac{\epsilon_{J}^{*} \cdot q}{m_{B}+m_{K_{J}^{*}}}\left[P^{\mu}-\frac{m_{B}^{2}-m_{K_{J}^{*}}^{2}}{q^{2}} q^{\mu}\right], \\
\left\langle K_{J}^{*}\left(P_{2}, \epsilon\right)\left|\bar{s} \sigma^{\mu \nu} q_{\nu} b\right| \bar{B}\left(P_{B}\right)\right\rangle= & -2 i T_{1}\left(q^{2}\right) \epsilon^{\mu \nu \rho \sigma} \epsilon_{J \nu}^{*} P_{B \rho} P_{2 \sigma}, \\
\left\langle K_{J}^{*}\left(P_{2}, \epsilon\right)\left|\bar{s} \sigma^{\mu \nu} \gamma_{5} q_{\nu} b\right| \bar{B}\left(P_{B}\right)\right\rangle= & T_{2}\left(q^{2}\right)\left[\left(m_{B}^{2}-m_{K_{J}^{*}}^{2}\right) \epsilon_{J \mu}^{*}-\epsilon_{J}^{*} \cdot q P^{\mu}\right]+T_{3}\left(q^{2}\right) \epsilon_{J}^{*} \cdot q\left[q^{\mu}-\frac{q^{2}}{m_{B}^{2}-m_{K_{J}^{*}}^{2}}\right],
\end{aligned}
$$

in which we have adopted $\epsilon^{0123}=+1, q=P_{B}-P_{2}$, and $P=P_{B}+P_{2}$. The polarization vector $\epsilon_{J}$ is constructed by the rank- $J$ polarization tensor

$$
\epsilon_{J \mu}(h)=\frac{1}{m_{B}^{J-1}} \epsilon_{\mu \nu_{1} \nu_{2} \ldots \nu_{J-1}}(h) P_{B}^{\nu_{1}} P_{B}^{\nu_{2}} \ldots P_{B}^{\nu_{J-1}},
$$

with the helicity $h=0, \pm 1$. In the case of $J=1$, it is reduced to the ordinary polarization vector.

These transition form factors have been calculated in the perturbative QCD approach [92 95] using the inputs from Ref. [96] and the results are summarized in Tab. II ${ }^{4}$, in which the dipole parametrization has been adopted to access

\footnotetext{
${ }^{4}$ Results for heavy-to-light form factors have been updated using a package that can compute form factors and two-body nonleptonic $B$ decays in the perturbative QCD approach available at: http://www.itkp.uni-bonn.de/ weiwang/
} 
TABLE II: $B \rightarrow K_{J}^{*}$ and $B_{s} \rightarrow f_{0}(980), \phi$ form factors in the perturbative QCD approach.

\begin{tabular}{|lccl|llll|}
\hline & $F(0)$ & $a_{F}$ & $b_{F}$ & & $F(0)$ & $a_{F}$ & $b_{F}$ \\
\hline$V^{\bar{B}^{0} \bar{K}^{* 0}}$ & 0.25 & -2.2 & 1.3 & $A_{0}^{\bar{B}^{0} \bar{K}^{* 0}}$ & 0.29 & -2.2 & 1.2 \\
$A_{1}^{\bar{B}^{0} \bar{K}^{* 0}}$ & 0.19 & -1.3 & 0.16 & $A_{2}^{\bar{B}^{0} \bar{K}^{* 0}}$ & 0.29 & -2.2 & 1.2 \\
$T_{1}^{\bar{B}^{0} \bar{K}^{* 0}}$ & 0.23 & -2.2 & 1.2 & $T_{2}^{\bar{B}^{0} \bar{K}^{* 0}}$ & 0.23 & -1.2 & 0.068 \\
$T_{3}^{\bar{B}^{0} \bar{K}^{* 0}}$ & 0.16 & -1.7 & 0.98 & & & & \\
$F_{1}^{\bar{B}^{0} \bar{K}_{0}^{* 0}(800)}$ & 0.27 & -2.1 & 1.2 & $F_{0}^{\bar{B}^{0} \bar{K}_{0}^{* 0}(800)}$ & 0.27 & -1.2 & 0.080 \\
$F_{T}^{\bar{B}^{0} \bar{K}_{0}^{* 0}(800)}$ & 0.30 & -2.2 & 1.2 & & & & \\
$V^{\bar{B}} \bar{B}_{s} \phi$ & 0.26 & -2.2 & 1.3 & $A_{0}^{\bar{B}_{s} \phi}$ & 0.30 & -2.2 & 1.2 \\
$A_{1}^{\bar{B}_{s} \phi}$ & 0.19 & -1.2 & 0.15 & $A_{2}^{\bar{B}_{s} \phi}$ & 0.30 & -2.2 & 1.2 \\
$T_{1}^{\bar{B}_{s} \phi}$ & 0.23 & -2.1 & 1.2 & $T_{2}^{\bar{B}_{s} \phi}$ & 0.23 & -1.2 & 0.066 \\
$T_{3}^{\bar{B}_{s} \phi}$ & 0.15 & -1.5 & 1.1 & & & & \\
$F_{1}^{\bar{B}_{s} f_{0}(980)}$ & 0.34 & -2.1 & 1.1 & $F_{0}^{\bar{B}_{s} f_{0}(980)}$ & 0.34 & -1.2 & 0.078 \\
$F_{T}^{\bar{B}_{s} f_{0}(980)}$ & 0.38 & -2.1 & 1.2 & & & & \\
\hline
\end{tabular}

the momentum transfer dependence

$$
F\left(q^{2}\right)=\frac{F(0)}{1+a_{F} q^{2} / m_{B}^{2}+b_{F}\left(q^{2} / m_{B}^{2}\right)^{2}} .
$$

Light-cone QCD sum rules results [97 100] have similar modulus and mass distributions for the transition form factors, and thus our discussion on semileptonic $B$ decays will not be affected.

\section{Appendix B: Helicity amplitudes}

The differential distributions can be expressed in terms of the helicity amplitude (see for instance Ref. [16]):

$$
\begin{aligned}
I_{1}^{c} & =\left(\left|A_{L 0}\right|^{2}+\left|A_{R 0}\right|^{2}\right)+8 \hat{m}_{l}^{2} \operatorname{Re}\left[A_{L 0} A_{R 0}^{*}\right]+4 \hat{m}_{l}^{2}\left|A_{t}\right|^{2}, \\
I_{1}^{s} & =\left(3 / 4-\hat{m}_{l}^{2}\right)\left[\left|A_{L \perp}\right|^{2}+\left|A_{L \| \mid}\right|^{2}+\left|A_{R \perp}\right|^{2}+\left|A_{R \| \mid}\right|^{2}\right]+4 \hat{m}_{l}^{2} \operatorname{Re}\left[A_{L \perp} A_{R \perp}^{*}+A_{L \|} A_{R \| \mid}^{*}\right] \\
I_{2}^{c} & =-\beta_{l}^{2}\left(\left|A_{L 0}\right|^{2}+\left|A_{R 0}\right|^{2}\right), \\
I_{2}^{s} & =\frac{1}{4} \beta_{l}^{2}\left(\left|A_{L \perp}\right|^{2}+\left|A_{L \|}\right|^{2}+\left|A_{R \perp}\right|^{2}+\left|A_{R \| \mid}\right|^{2}\right), \\
I_{3} & =\frac{1}{2} \beta_{l}^{2}\left(\left|A_{L \perp}\right|^{2}-\left|A_{L \|}\right|^{2}+\left|A_{R \perp}\right|^{2}-\left|A_{R \| \mid}\right|^{2}\right), \\
I_{4} & =\frac{1}{\sqrt{2}} \beta_{l}^{2}\left[\operatorname{Re}\left(A_{L 0} A_{L \|}^{*}\right)+\operatorname{Re}\left(A_{R 0} A_{R \| \mid}^{*}\right)\right], \quad I_{5}=\sqrt{2} \beta_{l}\left[\operatorname{Re}\left(A_{L 0} A_{L \perp}^{*}\right)-\operatorname{Re}\left(A_{R 0} A_{R \perp}^{*}\right)\right] \\
I_{6} & =2 \beta_{l}\left[\operatorname{Re}\left(A_{L \|} A_{L \perp}^{*}\right)-\operatorname{Re}\left(A_{R \|} A_{R \perp}^{*}\right)\right], \quad I_{7}=\sqrt{2} \beta_{l}\left[\operatorname{Im}\left(A_{L 0} A_{L \| \mid}^{*}\right)-\operatorname{Im}\left(A_{R 0} A_{R \| \mid}^{*}\right)\right] \\
I_{8} & =\frac{1}{\sqrt{2}} \beta_{l}^{2}\left[\operatorname{Im}\left(A_{L 0} A_{L \perp}^{*}\right)+\operatorname{Im}\left(A_{R 0} A_{R \perp}^{*}\right)\right], \quad I_{9}=\beta_{l}^{2}\left[\operatorname{Im}\left(A_{L \|} A_{L \perp}^{*}\right)+\operatorname{Im}\left(A_{R \| \mid} A_{R \perp}^{*}\right)\right]
\end{aligned}
$$


with $\hat{m}_{l}=m_{l} / \sqrt{q^{2}}$. Substituting the expressions $A_{i}$ into the angular coefficients, we obtain

$$
\begin{aligned}
& I_{1}^{c}=\sum_{J=0, \ldots}\left\{\left|Y_{J}^{0}\right|^{2}\left[\left|A_{L 0}^{J}\right|^{2}+\left|A_{R 0}^{J}\right|^{2}+8 \hat{m}_{l}^{2}\left|A_{L 0}^{J} A_{R 0}^{J *}\right| \cos \left(\delta_{L 0}^{J}-\delta_{R 0}^{J}\right)+4 \hat{m}_{l}^{2}\left|A_{t}^{J}\right|^{2}\right]\right. \\
& +\sum_{J^{\prime}=J+1, \ldots} Y_{J}^{0} Y_{J^{\prime}}^{0}\left[2 \cos \left(\delta_{L 0}^{J}-\delta_{L 0}^{J^{\prime}}\right)\left|A_{L 0}^{J}\right|\left|A_{L 0}^{J^{\prime} *}\right|+2 \cos \left(\delta_{R 0}^{J}-\delta_{R 0}^{J^{\prime}}\right)\left|A_{R 0}^{J}\right|\left|A_{R 0}^{J^{\prime} *}\right|\right. \\
& \left.\left.+8 \hat{m}_{l}^{2}\left[\cos \left(\delta_{L 0}^{J}-\delta_{R 0}^{J^{\prime}}\right)\left|A_{L 0}^{J} A_{R 0}^{J^{\prime} *}\right|+\cos \left(\delta_{L 0}^{J^{\prime}}-\delta_{R 0}^{J}\right)\left|A_{L 0}^{J^{\prime}} A_{R 0}^{J *}\right|\right]+8 \hat{m}_{l}^{2} \cos \left(\delta_{t}^{J}-\delta_{t}^{J^{\prime}}\right)\left|A_{t}^{J} \| A_{t}^{J^{\prime}}\right|\right]\right\}, \\
& I_{1}^{s}=\sum_{J=1, \ldots}\left\{| Y _ { J } ^ { - 1 } | ^ { 2 } \left[\left(3 / 4-\hat{m}_{l}^{2}\right)\left[\left|A_{L \perp}^{J}\right|^{2}+\left|A_{L \| \mid}^{J}\right|^{2}+\left|A_{R \perp}^{J}\right|^{2}+\left|A_{R \| \mid}^{J}\right|^{2}\right]\right.\right. \\
& \left.+4 \hat{m}_{l}^{2}\left(\cos \left(\delta_{L \perp}^{J}-\delta_{R \perp}^{J}\right)\left|A_{L \perp}^{J} A_{R \perp}^{J}\right|+\cos \left(\delta_{L \| \mid}^{J}-\delta_{R \| \mid}^{J}\right)\left|A_{L||}^{J} A_{R \|}^{J}\right|\right)\right] \\
& +\sum_{J^{\prime}=J+1, \ldots} Y_{J}^{-1} Y_{J^{\prime}}^{-1}\left[\left(3 / 4-\hat{m}_{l}^{2}\right)\left[2 \cos \left(\delta_{L \perp}^{J}-\delta_{L \perp}^{J^{\prime}}\right)\left|A_{L \perp}^{J} \| A_{L \perp}^{J^{\prime}}\right|+(L \rightarrow R)+(\perp \rightarrow \|)\right]\right. \\
& \left.\left.+4 \hat{m}_{l}^{2}\left[\cos \left(\delta_{L \perp}^{J}-\delta_{R \perp}^{J^{\prime}}\right)\left|A_{L \perp}^{J} A_{R \perp}^{J^{\prime} *}\right|+\cos \left(\delta_{L \perp}^{J^{\prime}}-\delta_{R \perp}^{J}\right)\left|A_{L \perp}^{J^{\prime}} A_{R \perp}^{J *}\right|+(\perp \rightarrow||)\right]\right]\right\}, \\
& I_{2}^{c}=-\beta_{l}^{2} \sum_{J=0, \ldots}\left\{\left|Y_{J}^{0}\right|^{2}\left(\left|A_{L 0}^{J}\right|^{2}+\left|A_{R 0}^{J}\right|^{2}\right)+\sum_{J^{\prime}=J+1, \ldots} Y_{J}^{0} Y_{J^{\prime}}^{0}\left[2 \cos \left(\delta_{L 0}^{J}-\delta_{L 0}^{J^{\prime}}\right)\left|A_{L 0}^{J}\right|\left|A_{L 0}^{J^{\prime}}\right|+(L \rightarrow R)\right]\right\}, \\
& I_{2}^{s}=\frac{1}{4} \beta_{l}^{2} \sum_{J=0, \ldots}\left\{\left|Y_{J}^{-1}\right|^{2}\left[\left(\left|A_{L \perp}^{J}\right|^{2}+\left|A_{L||}^{J}\right|^{2}\right)\right]\right. \\
& +\sum_{J^{\prime}=J+1} Y_{J}^{-1} Y_{J^{\prime}}^{-1}\left[2 \cos \left(\delta_{L \perp}^{J}-\delta_{L \perp}^{J^{\prime}}\right)\left|A_{L \perp}^{J}\left\|A_{L \perp}^{J^{\prime}}\left|+2 \cos \left(\delta_{L \|}^{J}-\delta_{L||}^{J^{\prime}}\right)\right| A_{L\|\|}^{J}\right\| A_{L\|\|}^{J^{\prime}} \|\right]+(L \rightarrow R)\right\}, \\
& I_{3}=\frac{1}{2} \beta_{l}^{2} \sum_{J=1, \ldots}\left\{\left|Y_{J}^{-1}\right|^{2}\left[\left(\left|A_{L \perp}^{J}\right|^{2}-\left|A_{L||}^{J}\right|^{2}\right)\right]\right. \\
& \left.+\sum_{J^{\prime}=J+1} Y_{J}^{-1} Y_{J^{\prime}}^{-1}\left[2 \cos \left(\delta_{L \perp}^{J}-\delta_{L \perp}^{J^{\prime}}\right)\left|A_{L \perp}^{J}\left\|A_{L \perp}^{J^{\prime}}\left|-2 \cos \left(\delta_{L \|}^{J}-\delta_{L||}^{J^{\prime}}\right)\right| A_{L \| \mid}^{J}\right\| A_{L\|\|}^{J^{\prime}}\right|\right]+(L \rightarrow R)\right\}, \\
& I_{4}=\frac{1}{\sqrt{2}} \beta_{l}^{2} \sum_{J=1, \ldots} \sum_{J^{\prime}=1, . .}\left[Y_{J}^{0} Y_{J^{\prime}}^{-1}\left|A_{L 0}^{J} A_{L \|}^{J^{\prime} *}\right| \cos \left(\delta_{L 0}^{J}-\delta_{L \|}^{J^{\prime}}\right)+(L \rightarrow R)\right] \\
& I_{5}=\sqrt{2} \beta_{l} \sum_{J=0, \ldots} \sum_{J^{\prime}=1, . .}\left[Y_{J}^{0} Y_{J^{\prime}}^{-1}\left|A_{L 0}^{J} A_{L \perp}^{J^{\prime} *}\right| \cos \left(\delta_{L 0}^{J}-\delta_{L \perp}^{J^{\prime}}\right)-(L \rightarrow R)\right], \\
& I_{6}=2 \beta_{l} \sum_{J=1, \ldots}\left\{\left|Y_{J}^{-1}\right|^{2}\left|A_{L||}^{J} A_{L \perp}^{J *}\right| \cos \left(\delta_{L||}^{J}-\delta_{L \perp}^{J}\right)\right. \\
& \left.+\sum_{J^{\prime}=J+1} Y_{J}^{-1} Y_{J^{\prime}}^{-1}\left[\cos \left(\delta_{L||}^{J}-\delta_{L \perp}^{J^{\prime}}\right)\left|A_{L||}^{J} A_{L \perp}^{J^{\prime}}\right|+\cos \left(\delta_{L||}^{J^{\prime}}-\delta_{L \perp}^{J}\right)\left|A_{L||}^{J^{\prime}} A_{L \perp}^{J}\right|\right]-(L \rightarrow R)\right\}, \\
& I_{7}=\sqrt{2} \beta_{l} \sum_{J=0, \ldots} \sum_{J^{\prime}=1, . .}\left[Y_{J}^{0} Y_{J^{\prime}}^{-1}\left|A_{L 0}^{J} A_{L \| \mid}^{J^{\prime} *}\right| \sin \left(\delta_{L 0}^{J}-\delta_{L \| \mid}^{J^{\prime}}\right)-(L \rightarrow R)\right], \\
& I_{8}=\frac{1}{\sqrt{2}} \beta_{l}^{2} \sum_{J=0, \ldots} \sum_{J^{\prime}=1, . .}\left[Y_{J}^{0} Y_{J^{\prime}}^{-1}\left|A_{L 0}^{J} A_{L \perp}^{J^{\prime} *}\right| \sin \left(\delta_{L 0}^{J}-\delta_{L \perp}^{J^{\prime}}\right)+(L \rightarrow R)\right] \\
& I_{9}=\beta_{l}^{2} \sum_{J=1, \ldots}\left\{\left|Y_{J}^{-1}\right|^{2}\left|A_{L||}^{J} A_{L \perp}^{J}\right| \sin \left(\delta_{L||}^{J}-\delta_{L \perp}^{J}\right)\right. \\
& \left.+\sum_{J^{\prime}=J+1} Y_{J}^{-1} Y_{J^{\prime}}^{-1}\left[\sin \left(\delta_{L \|}^{J}-\delta_{L \perp}^{J^{\prime}}\right)\left|A_{L||}^{J} A_{L \perp}^{J^{\prime}}\right|+\sin \left(\delta_{L \|}^{J^{\prime}}-\delta_{L \perp}^{J}\right)\left|A_{L||}^{J^{\prime}} A_{L \perp}^{J}\right|\right]+(L \rightarrow R)\right\},
\end{aligned}
$$

where for brevity we have omitted the argument in the spherical harmonics functions: $Y_{J}^{i} \equiv Y_{J}^{i}\left(\theta_{K}, 0\right)$. Transverse amplitudes vanish for $J=0$ since spin-0 mesons have only one polarization configuration. 


\section{Appendix C: Normalization of the Line-Shape}

Assuming the matrix elements to be saturated by resonances, we have

$$
\left\langle K \pi|[\bar{s} b]| \bar{B}^{0}\right\rangle \simeq \int d^{4} p_{K_{J}^{*}} \frac{\left\langle K \pi \mid K_{J}^{*}\right\rangle\left\langle K_{J}^{*}|[\bar{s} b]| \bar{B}^{0}\right\rangle}{p_{K_{J}^{*}}^{2}-m_{K_{J}^{*}}^{2}+i m_{K_{J}^{*}} \Gamma_{K_{J}^{*}}} \sim \frac{\left\langle K_{J}^{*}|[\bar{s} b]| \bar{B}^{0}\right\rangle\langle K \pi|\bar{s} u| 0\rangle}{\left\langle K_{J}^{*}|\bar{s} u| 0\right\rangle}
$$

and

$$
\langle K \pi|\bar{s} u| 0\rangle \simeq \int d^{4} p_{K_{J}^{*}} \frac{\left\langle K \pi \mid K_{J}^{*}\right\rangle\left\langle K_{J}^{*}|\bar{s} u| 0\right\rangle}{p_{K_{J}^{*}}^{2}-m_{K_{J}^{*}}^{2}+i m_{K_{J}^{*}} \Gamma_{K_{J}^{*}}}
$$

By comparing these two equations, we derive the line-shape used in this work:

$$
L_{K_{0}^{*}}\left(m_{K \pi}\right)=\sqrt{\frac{|\vec{p}|}{8 \pi m_{K_{0}^{*}}}} \frac{\langle K \pi|\bar{s} u| 0\rangle}{\left\langle K_{0}^{*}|\bar{s} u| 0\right\rangle} .
$$

Although this equation can not be taken literally, we determine the normalization constant $\mathcal{N}_{\chi \mathrm{PT}}$ from this equation, bearing in mind the large uncertainties. It is important to notice that below threshold the momentum becomes negative and thus an extra imaginary part is introduced. This is not appropriate in the case of $K \bar{K}$, and thus we use the Flatté model instead.

[1] J. P. Lees et al. [BaBar Collaboration], Phys. Rev. D 86, 032012 (2012) arXiv:1204.3933 [hep-ex]].

[2] J.-T. Wei et al. [BELLE Collaboration], Phys. Rev. Lett. 103, 171801 (2009) arXiv:0904.0770 [hep-ex]].

[3] T. Aaltonen et al. [CDF Collaboration], Phys. Rev. Lett. 106, 161801 (2011) arXiv:1101.1028 [hep-ex]].

[4] R. Aaij et al. [LHCb Collaboration], arXiv:1304.6325 [hep-ex].

[5] A. Ali, P. Ball, L. T. Handoko and G. Hiller, Phys. Rev. D 61, 074024 (2000) hep-ph/9910221.

[6] C.-H. Chen and C. Q. Geng, Nucl. Phys. B 636, 338 (2002) hep-ph/0203003.

[7] F. Krüger and J. Matias, Phys. Rev. D 71, 094009 (2005) hep-ph/0502060.

[8] U. Egede, T. Hurth, J. Matias, M. Ramon and W. Reece, JHEP 0811, 032 (2008) arXiv:0807.2589 [hep-ph]].

[9] W. Altmannshofer, P. Ball, A. Bharucha, A. J. Buras, D. M. Straub and M. Wick, JHEP 0901, 019 (2009) arXiv:0811.1214 [hep-ph]].

[10] C.-W. Chiang, R.-H. Li and C.-D. Lu, Chin. Phys. C 36, 14 (2012) arXiv:0911.2399 [hep-ph]].

[11] A. Khodjamirian, T. .Mannel, A. A. Pivovarov and Y. -M. Wang, JHEP 1009, 089 (2010) arXiv:1006.4945 [hep-ph]].

[12] C. Bobeth, G. Hiller and D. van Dyk, JHEP 1107, 067 (2011) arXiv:1105.0376 [hep-ph]].

[13] C. Bobeth, G. Hiller and D. van Dyk, Phys. Rev. D 87, 034016 (2013) arXiv:1212.2321 [hep-ph]].

[14] S. Jäger and J. M. Camalich, JHEP 1305, 043 (2013) arXiv:1212.2263 [hep-ph]].

[15] S. Descotes-Genon, T. Hurth, J. Matias and J. Virto, JHEP 1305, 137 (2013) arXiv:1303.5794 [hep-ph]].

[16] C.-D. Lu and W. Wang, Phys. Rev. D 85, 034014 (2012) arXiv:1111.1513 [hep-ph]].

[17] R.-H. Li, C.-D. Lu and W. Wang, Phys. Rev. D 83, 034034 (2011) arXiv:1012.2129 [hep-ph]].

[18] D. Becirevic and A. Tayduganov, Nucl. Phys. B 868, 368 (2013) arXiv:1207.4004 [hep-ph]].

[19] J. Matias, Phys. Rev. D 86, 094024 (2012) arXiv:1209.1525 [hep-ph]].

[20] T. Blake, U. Egede and A. Shires, JHEP 1303, 027 (2013) arXiv:1210.5279 [hep-ph]].

[21] P. del Amo Sanchez et al. [BaBar Collaboration], Phys. Rev. D 83, 072001 (2011) [arXiv:1012.1810 [hep-ex]].

[22] J. M. Link et al. [FOCUS Collaboration], Phys. Lett. B 681, 14 (2009) arXiv:0905.4846 [hep-ex]].

[23] E. M. Aitala et al. [E791 Collaboration], Phys. Rev. D 73, 032004 (2006) [Erratum-ibid. D 74, 059901 (2006)] hep-ex/0507099.

[24] J. A. Oller, Phys. Rev. D 71, 054030 (2005) hep-ph/0411105.

[25] I. Bediaga and J. M. de Miranda, Phys. Lett. B 633, 167 (2006) hep-ex/0405019. 
[26] P. C. Magalhaes, M. R. Robilotta, K. S. F. F. Guimaraes, T. Frederico, W. de Paula, I. Bediaga, A. C. d. Reis and C. M. Maekawa et al., Phys. Rev. D 84, 094001 (2011) arXiv:1105.5120 [hep-ph]].

[27] H. Kamano, S. X. Nakamura, T. S. H. Lee and T. Sato, Phys. Rev. D 84, 114019 (2011) [arXiv:1106.4523 [hep-ph]].

[28] D. V. Bugg, Eur. Phys. J. A 25, 107 (2005) [Erratum-ibid. A 26, 151 (2005)] hep-ex/0510026.

[29] D. Aston, N. Awaji, T. Bienz, F. Bird, J. D'Amore, W. M. Dunwoodie, R. Endorf and K. Fujii et al., Nucl. Phys. B 296, 493 (1988).

[30] C. B. Lang, L. Leskovec, D. Mohler and S. Prelovsek, Phys. Rev. D 86, 054508 (2012) arXiv:1207.3204 [hep-lat]].

[31] S. R. Beane, P. F. Bedaque, T. C. Luu, K. Orginos, E. Pallante, A. Parreno and M. J. Savage, Phys. Rev. D 74, 114503 (2006) hep-lat/0607036.

[32] M. Döring and U.-G. Meißner, JHEP 1201, 009 (2012) arXiv:1111.0616 [hep-lat]].

[33] M. Döring, U.-G. Meißner, E. Oset and A. Rusetsky, Eur. Phys. J. A 48, 114 (2012) arXiv:1205.4838 [hep-lat]].

[34] M. Döring, U.-G. Meißner, E. Oset and A. Rusetsky, Eur. Phys. J. A 47, 139 (2011) arXiv:1107.3988 [hep-lat]].

[35] B. Aubert et al. [BaBar Collaboration], Phys. Rev. D 78, 051101 (2008) arXiv:0807.1599 [hep-ex]].

[36] K. M. Ecklund et al. [CLEO Collaboration], Phys. Rev. D 80, 052009 (2009) arXiv:0907.3201 [hep-ex]].

[37] S. Gardner and U.-G. Meißner, Phys. Rev. D 65, 094004 (2002) hep-ph/0112281.

[38] Y.-Y. Keum, H.-N. Li and A. I. Sanda, Phys. Lett. B 504, 6 (2001) hep-ph/0004004.

[39] Y. Y. Keum, H.-N. Li and A. I. Sanda, Phys. Rev. D 63, 054008 (2001) hep-ph/0004173.

[40] C.-D. Lu, K. Ukai and M.-Z. Yang, Phys. Rev. D 63, 074009 (2001) hep-ph/0004213.

[41] C.-D. Lu and M.-Z. Yang, Eur. Phys. J. C 23, 275 (2002) hep-ph/0011238.

[42] H.-N. Li, Y.-L. Shen and Y.-M. Wang, Phys. Rev. D 85, 074004 (2012) arXiv:1201.5066 [hep-ph]].

[43] J. Beringer et al. [Particle Data Group Collaboration], Phys. Rev. D 86, 010001 (2012).

[44] J. Gasser and U.-G. Meißner, Nucl. Phys. B 357, 90 (1991).

[45] U.-G. Meißner and J. A. Oller, Nucl. Phys. A 679, 671 (2001) hep-ph/0005253.

[46] J. A. Oller, E. Oset and J. E. Palomar, Phys. Rev. D 63, 114009 (2001) hep-ph/0011096.

[47] M. Frink, B. Kubis and U.-G. Meißner, Eur. Phys. J. C 25, 259 (2002) hep-ph/0203193.

[48] J. Bijnens and P. Talavera, Nucl. Phys. B 669, 341 (2003) hep-ph/0303103.

[49] T. A. Lähde and U.-G. Meißner, Phys. Rev. D 74, 034021 (2006) hep-ph/0606133.

[50] V. Bernard and E. Passemar, JHEP 1004, 001 (2010) arXiv:0912.3792 [hep-ph]].

[51] Z.-H. Guo, J. A. Oller and J. Ruiz de Elvira, Phys. Rev. D 86, 054006 (2012) arXiv:1206.4163 [hep-ph]].

[52] J. F. Donoghue, J. Gasser and H. Leutwyler, Nucl. Phys. B 343, 341 (1990).

[53] M. Jamin, J. A. Oller and A. Pich, Nucl. Phys. B 587, 331 (2000) hep-ph/0006045.

[54] M. Jamin, J. A. Oller and A. Pich, Nucl. Phys. B 622, 279 (2002) hep-ph/0110193.

[55] M. Jamin, J. A. Oller and A. Pich, Phys. Rev. D 74, 074009 (2006) hep-ph/0605095.

[56] V. Bernard and E. Passemar, Phys. Lett. B 661, 95 (2008) arXiv:0711.3450 [hep-ph]].

[57] F. Guerrero and A. Pich, Phys. Lett. B 412, 382 (1997) hep-ph/9707347.

[58] F.-K. Guo, C. Hanhart, F. J. Llanes-Estrada and U.-G. Meißner, Phys. Lett. B 678, 90 (2009) arXiv:0812.3270 [hep-ph]].

[59] C. Hanhart, Phys. Lett. B 715, 170 (2012) arXiv:1203.6839 [hep-ph]].

[60] V. Bernard, D. R. Boito and E. Passemar, Nucl. Phys. Proc. Suppl. 218, 140 (2011) arXiv:1103.4855 [hep-ph]].

[61] J. A. Oller, E. Oset and J. R. Pelaez, Phys. Rev. D 59, 074001 (1999) [Erratum-ibid. D 60, 099906 (1999)] [Erratum-ibid. D 75, 099903 (2007)] hep-ph/9804209.

[62] Z.-H. Guo and J. A. Oller, Phys. Rev. D 84, 034005 (2011) arXiv:1104.2849 [hep-ph]].

[63] M. Albaladejo and J. A. Oller, Phys. Rev. Lett. 101, 252002 (2008) arXiv:0801.4929 [hep-ph]].

[64] A. Gomez Nicola and J. R. Pelaez, Phys. Rev. D 65, 054009 (2002) hep-ph/0109056.

[65] V. Bernard, N. Kaiser and U.-G. Meißner, Nucl. Phys. B 364 (1991) 283.

[66] S. Descotes-Genon and B. Moussallam, Eur. Phys. J. C 48, 553 (2006) hep-ph/0607133.

[67] D. V. Bugg, Phys. Rev. D 81, 014002 (2010) arXiv:0906.3992 [hep-ph]].

[68] P. Büttiker, S. Descotes-Genon and B. Moussallam, Eur. Phys. J. C 33, 409 (2004) hep-ph/0310283.

[69] S. Descotes-Genon, Eur. Phys. J. C 52, 141 (2007) hep-ph/0703154 [HEP-PH]].

[70] J. Gasser and H. Leutwyler, Nucl. Phys. B 250, 517 (1985). 
[71] J. Bijnens and I. Jemos, Nucl. Phys. B 854, 631 (2012) arXiv:1103.5945 [hep-ph]].

[72] J. Gasser and H. Leutwyler, Nucl. Phys. B 250, 465 (1985).

[73] F. Niecknig, B. Kubis and S. P. Schneider, Eur. Phys. J. C 72, 2014 (2012) [arXiv:1203.2501 [hep-ph]].

[74] M. I. Haftel and F. Tabakin, Nucl. Phys. A 158, 1 (1970).

[75] M. Hoferichter, C. Ditsche, B. Kubis and U.-G. Meißner, JHEP 1206, 063 (2012) [arXiv:1204.6251 [hep-ph]].

[76] J. A. Oller and L. Roca, Phys. Lett. B 651, 139 (2007) arXiv:0704.0039 [hep-ph]].

[77] J. Gasser and H. Leutwyler, Annals Phys. 158, 142 (1984).

[78] J. A. Oller and E. Oset, Nucl. Phys. A 620, 438 (1997) [Erratum-ibid. A 652, 407 (1999)] hep-ph/9702314.

[79] B. Aubert et al. [BaBar Collaboration], Phys. Rev. D 72, 072003 (2005) [Erratum-ibid. D 74, 099903 (2006)] hep-ex/0507004.

[80] B. Aubert et al. [BaBar Collaboration], Phys. Rev. D 79, 112001 (2009) arXiv:0811.0564 [hep-ex]].

[81] S. M. Flatté, Phys. Lett. B 63, 224 (1976).

[82] M. Ablikim et al. [BES Collaboration], Phys. Lett. B 607, 243 (2005) hep-ex/0411001].

[83] RAaij et al. [ LHCb Collaboration], arXiv:1305.2168 [hep-ex].

[84] J. Charles, A. Le Yaouanc, L. Oliver, O. Pene and J. C. Raynal, Phys. Rev. D 60, 014001 (1999) hep-ph/9812358.

[85] M. Diehl, A. Manashov and A. Schäfer, Phys. Lett. B 622, 69 (2005) hep-ph/0505269.

[86] C.-H. Chen and H.-N. Li, Phys. Lett. B 561, 258 (2003) hep-ph/0209043.

[87] H.-Y. Cheng, C.-K. Chua and A. Soni, Phys. Rev. D 72, 094003 (2005) hep-ph/0506268.

[88] B. El-Bennich, A. Furman, R. Kaminski, L. Lesniak, B. Loiseau and B. Moussallam, Phys. Rev. D 79, 094005 (2009) [Erratum-ibid. D 83, 039903 (2011)] arXiv:0902.3645 [hep-ph]].

[89] H. Hatanaka and K.-C. Yang, Eur. Phys. J. C 67, 149 (2010) arXiv:0907.1496 [hep-ph]].

[90] W. Wang, Phys. Rev. D 83, 014008 (2011) arXiv:1008.5326 [hep-ph]].

[91] K.-C. Yang, Phys. Lett. B 695, 444 (2011) [arXiv:1010.2944 [hep-ph]].

[92] R.-H. Li, C.-D. Lu, W. Wang and X.-X. Wang, Phys. Rev. D 79, 014013 (2009) arXiv:0811.2648 [hep-ph]].

[93] W. Wang, Y.-L. Shen, Y. Li and C.-D. Lu, Phys. Rev. D 74, 114010 (2006) hep-ph/0609082.

[94] Y.-L. Shen, W. Wang, J. Zhu and C.-D. Lu, Eur. Phys. J. C 50, 877 (2007) hep-ph/0610380.

[95] C. S. Kim, Y. Li and W. Wang, Phys. Rev. D 81, 074014 (2010) arXiv:0912.1718 [hep-ph]].

[96] H.-Y. Cheng, C.-K. Chua and K.-C. Yang, Phys. Rev. D 73, 014017 (2006) hep-ph/0508104.

[97] P. Ball and R. Zwicky, Phys. Rev. D 71, 014029 (2005) hep-ph/0412079.

[98] A. Khodjamirian, T. Mannel and N. Offen, Phys. Rev. D 75, 054013 (2007) hep-ph/0611193.

[99] P. Colangelo, F. De Fazio and W. Wang, Phys. Rev. D 81, 074001 (2010) arXiv:1002.2880 [hep-ph]].

[100] P. Colangelo, F. De Fazio and W. Wang, Phys. Rev. D 83, 094027 (2011) arXiv:1009.4612 [hep-ph]]. 